\title{
A Heterogeneous Reliable Location Model with Risk Pooling under Supply Disruptions
}

\begin{abstract}
This paper investigates a facility location model that considers the disruptions of facilities and the cost savings from the inventory risk-pooling effect and economies of scale. Facilities may have heterogenous disruption probabilities. When a facility fails, its customers may be reassigned to other surviving ones to hedge against lost-sales costs. We first develop both an exact and an approximate expression for the nonlinear inventory cost, and then formulate the problem as a nonlinear integer programming model. The objective is to minimize the expected total cost across all possible facility failure scenarios. To solve this problem, we design two methods, an exact approach using special ordered sets of type two (SOS2) and a heuristic based on Lagrangian relaxation. We test the model and algorithms on data sets with up to 150 nodes. Computational results show that the proposed algorithms can solve the problem efficiently in reasonable time. Managerial insights on the optimal facility deployment, customer assignments and inventory control strategies are also drawn.
\end{abstract}

Keywords: facility location; disruption; risk pooling; concave minimization; Lagrangian relaxation

\section{Introduction}

This paper introduces the heterogeneous reliable location model with risk pooling (HRLMRP), a facility location model that considers the disruptions of facilities and the cost savings from the inventory riskpooling effect and economies of scale. It has been observed that facility location models that consider inventory costs tend to produce solutions with fewer open facilities than the solutions to classical location models, due to the concave nature of the inventory requirements and the subsequent incentive for physical pooling (Shen et al.|2003). On the other hand, the risk of disruptions leads to solutions that have more open facilities than classical models, due to the so-called risk-diversification effect (Snyder) and Daskin 2005). By considering both inventory and disruptions, our facility location model seeks the appropriate balance between these two competing tendencies.

We consider a three-tiered supply chain network that consists of a single supplier, multiple distribution centers (DCs $\mathrm{s}^{1}$ ) and customers. Customers have uncorrelated probabilistic demands that follow a normal distribution and are satisfied by the supplier. DCs combine the orders from different customers and then order from the supplier. Each DC maintains safety stocks to ensure a pre-specific level of

\footnotetext{
${ }^{1}$ We use "DC" and "facility" interchangeably.
} 
service. The inventory costs in our model capture both the "risk pooling effect" (Eppen 1979), which says that the cost of a centralized inventory is less than the sum of the costs of decentralized inventories, as well as economies of scale, which are explicitly reflected by a fixed cost term.

In addition, DCs are subject to disruptions, which may result from natural disasters, labor actions, etc. When a DC is disrupted, it cannot serve customers. Following Snyder and Daskin (2005), we assume that customers of disrupted DCs are reassigned to another, functional, DC. In particular, we assign each customer to multiple DCs in a sequence of assignment levels $r=0,1,2, \cdots$. For each customer, the lowest-level DC $(r=0)$, the so-called the primary DC, will serve it under normal circumstances. If the primary DC fails, the customer is served by its first backup DC $(r=1)$. If that DC fails too, it is served by its level-2 DC, and so on. In general, a DC assigned to a customer at level $r$ serves that customer if and only if all $r$ DCs at lower levels have failed. If all of the DCs assigned to the customer are disrupted, or when the total cost of satisfying the customer's demands is higher than lost sales cost, the customer is not served and a penalty cost is imposed.

This problem is formulated as a mixed integer nonlinear programming (MINLP) model. The model determines the number and locations of DCs, the assignment of customers to DCs at all levels, and the approximate optimal ordering policy at the DCs in order to minimize the expected total cost (across all possible facility disruption scenarios), which includes: (1) the fixed cost to locate DCs, (2) the shipment cost from the supplier to the located DCs and from the DCs to the customers, (3) the working inventory cost and safety stock cost at the located DCs, and (4) the lost sales cost. Our problem is NP-hard. To solve it, we design two methods, an exact approach using special ordered sets of type two (SOS2) and a Lagrangian relaxation heuristic. We test both algorithms using benchmark instances with 49, 88, and 150 nodes based on U.S. census data.

Our model is among the first to consider both inventory costs and disruptions in the context of a facility location problem, thus balancing the competing tendencies toward consolidation and diversification. Moreover, ours is the first paper to consider site-dependent disruption probabilities, which add considerable realism but also computational complexity, in this context.

The remainder of this paper is organized as follows. We review the relevant literature in \$2, and formulate the HRLMRP as a MINLP in $\$$. An exact approach using SOS2 and a heuristic using Lagrangian relaxation are provided in $\$ 4$ and $\$ 5$, respectively, followed by the numerical experiment design and computational results in $\$ 6$. Finally, $\$ 7$ concludes the paper and discusses future research. 


\section{Literature Review}

Facility location problems have been extensively studied in the past few decades, due to their wide applications in numerous contexts such as supply chain planning, public service systems, and transportation infrastructure deployment. One of the most well-studied problems is the uncapacitated fixed-charge location problem (UFLP), which seeks the optimal facility locations and customer assignments to balance the tradeoff between initial setup costs and day-to-day shipment costs. For overviews of this classical model, see Snyder and Shen (2011), Daskin (2013).

In the UFLP, an implicit assumption is that all the facilities are always available. In contrast, Snyder and Daskin (2005) introduce the reliable fixed-charge location problem (RFLP), in which facilities are subject to random disruptions with equal probability, to investigate the effect of probabilistic facility failures on the optimal facility deployment. They also propose techniques for evaluating the cost/service trade-off. Berman et al. (2007), Shen et al. (2011), Cui et al. (2010) and Aboolian et al. (2012) relax the assumption of identical failure probabilities. Berman et al. (2007) focus on asymptotic properties of the problem, along with heuristics that have worst-case performance bounds. Shen et al. (2011) formulate the RFLP as a nonlinear integer program and propose approximation algorithms for the special case with identical probabilities. Cui et al. (2010) formulate the RFLP with site-dependent failure probabilities as a nonlinear mixed integer program and then apply a linearization technique. They propose a Lagrangian relaxation algorithm, which is efficient if the maximum number of facilities assigned to a customer is relative small. Aboolian et al. (2012) study the same problem as Cui et al. (2010), but they do not limit the number of facilities assigned to a customer. They develop exact and approximate algorithms, based on finding successive lower and upper bounds. See Snyder et al. (2015) for a review of the literature on supply chain management (including facility location) with disruptions.

Inventory costs are not included in either the UFLP or RFLP. As a result, applying UFLP-type models to networks with non-trivial inventory costs may lead to suboptimal design and erroneous system cost estimation. Daskin et al. (2002) and Shen et al. (2003) introduce the location model with risk pooling (LMRP), which incorporates both transportation and inventory costs in a facility location context. Doing so requires nonconvex terms in the objective function, and much of the literature on this topic has focused on solving this problem (and its descendants) efficiently, despite the nonconvexity. For example, Daskin et al. (2002) propose a Lagrangian relaxation algorithm, while Shen et al. (2003) present a column generation approach. Both assume that the variance-to-mean ratio is identical for all customers, which allows the number of nonlinear terms per DC to be reduced from two to one. 
Shu et al. (2005) relax this assumption and propose a somewhat less efficient algorithm based on computational geometry. Other authors have proposed extensions of the LMRP. For example, Ozsen et al. (2008) introduce the capacitated LMRP (CLMRP), in which each DC has limited capacity, Ozsen et al. (2009) analyze the effect of multisourcing on the CLMRP, and Shen and Qi (2007) incorporate routing decisions; all of these articles propose algorithms based on Lagrangian relaxation. The recent paper by Maass et al. (2015) introduces inventory into the classical capacitated fixed-charge location problem (CFLP). However, in their model, inventory is used as a tool to allow violations of the capacity constraints, whereas in the LMRP it is an inherent part of the cost function.

These location-inventory models assume that facilities are always available, that is, that the supply process is deterministic. More recently, several papers have studied location-inventory models with supply disruptions. Qi and Shen (2007) model random disruptions at the DCs, while Qi et al. (2010) allow disruptions to occur at either the supplier or the customers. Both studies use inventory to mitigate the disruptions and do not allow customers to be reassigned after a disruption, as in the RFLP and our model. In contrast, Jeon (2008), Aryanezhad et al. (2010) and Chen et al. (2011) study the reliable LMRP (RLMRP) and allow customers of disrupted facilities to be served by backup facilities. The dissertation by Jeon (2008) proposes two methods, a Lagrangian relaxation algorithm with a piecewise linear approximation and an approach using SOS2. Aryanezhad et al. (2010) develop a solution approach based on genetic algorithms. Chen et al. (2011) design a Lagrangian relaxation solution framework for the problem, including a polynomial-time exact algorithm for a relaxation of the nonlinear subproblem. However, all of these studies assume that the disruption probability is the same at every facility, an assumption that we relax in this paper.

Our model can be considered as an extension of the three reliable location-inventory studies discussed above, in which we relax the assumption of identical failure probabilities. We first formulate the HRLMRP as a nonlinear mixed integer programming model, and then apply the linearization technique used by Cui et al. (2010), as well as SOS2, to linearize the formulation. The resulting model can be solved exactly using a commercial MIP solver. In addition, we design a heuristic based on Lagrangian relaxation. We conduct an extensive numerical study to test the solution approaches and draw insights on the optimal facility deployment.

The main contributions of this paper are as follows: First, we develop an exact expression as well as a high-accuracy approximate expression for the expected inventory cost under heterogenous failure probabilities. Second, we construct a new nonlinear mixed integer formulation for the joint locationinventory problem with both facility disruptions and demand uncertainty, and apply linearization 
techniques and SOS2 to find exact solutions. Third, we develop a customized Lagrangian relaxation heuristic based on finding successive lower and upper bounds.

\section{Model Formulation}

In this section, we first introduce the notation that will be used throughout the paper. We then derive both the exact and approximate expressions for the inventory cost. Finally, we formulate the problem as a nonlinear programming model.

\subsection{Notation}

\subsubsection{Parameters}

Define $I$ to be the set of customers, indexed by $i$, and $J$ to be the set of candidate facility locations, indexed by $j$. We assume that the probability that facility $j$ is disrupted at any instant in time is given by $0 \leq q_{j}<1$, and that facility disruptions are independent of each other. When a facility fails, it cannot provide any service and its original customers will be either diverted to other surviving facilities or subject to a certain penalty. We further assume that each customer is assigned to up to $R$ facilities (where $R$ is a constant, $1 \leq R \leq|J|$ ) and can be serviced by these and only these facilities.

Assume that the demand at each customer $i \in I$ is normally distributed with a daily mean of $\mu_{i}$

and a daily variance of $\sigma_{i}^{2}$. As in Daskin et al. (2002), we also assume that $\sigma_{i}^{2} / \mu_{i}=\gamma, \forall i \in I$, for a constant $\gamma \geq 0$. The cost per unit of demand to ship from facility $j \in J$ to customer $i \in I$ is given by $c_{i j}$. Associated with each customer $i$ is a cost $\pi_{i}$ that represents the cost of not serving the customer, per unit of demand. To model this, we add a dummy facility $e$ that has fixed cost $f_{e}=0$, failure probability $q_{e}=0$, and unit transportation cost $c_{i e}=\pi_{i}$ for all customers $i \in I$. Let $J_{e}=J \cup\{e\}$.

Placing an order from facility $j$ to the supplier incurs a fixed ordering $\operatorname{cost} F_{j}$, a fixed transportation $\operatorname{cost} g_{j}$, and a volume-dependent transportation cost $a_{j}$ per unit. These replenishment orders arrive after a lead time of $L$ days. On-hand inventory at facility $j$ incurs a holding cost of $h_{j}$ per unit per day. The transportation and inventory cost components of the objective function are assigned weights of $\beta$ and $\theta$, respectively; or these can both be set to 1 if the units are commensurate.

To summarize, we have the following parameters:

- $I=$ set of customers, indexed by $i$;

- $J=$ set of candidate DC sites, indexed by $j$; 
- $f_{j}=$ fixed cost of locating a DC at candidate site $j$;

- $F_{j}=$ fixed cost of placing an order from DC $j$ to the supplier;

- $g_{j}, a_{j}=$ fixed cost and per-unit cost of a shipment from the supplier to DC $j$;

- $q_{j}=$ failure probability of DC $j$;

- $h_{j}=$ unit holding cost of DC $j$, per unit per day;

- $\mu_{i}=$ daily mean demand at customer $i$;

- $\sigma_{i}^{2}=$ daily variance of demand at customer $i$;

- $c_{i j}=$ cost per unit shipped to customer $i$ from candidate DC site $j$;

- $L=$ lead time (in days) for deliveries from the supplier to the DCs;

- $z_{\alpha}=$ standard normal deviate such that $P\left(Z \leq z_{\alpha}\right)=\alpha$;

- $\beta, \theta=$ weights assigned to transportation and inventory costs, respectively;

\subsubsection{Decision Variables}

The variables used in this model are the location variables $(\boldsymbol{y})$, the assignment variables $(\boldsymbol{z})$ and the probability variables $(\boldsymbol{p})$.

$$
\begin{aligned}
& y_{j}= \begin{cases}1, & \text { if facility } j \text { is open, } \\
0, & \text { otherwise }\end{cases} \\
& z_{i j r}= \begin{cases}1, & \text { if customer } i \text { is assigned to facility } j \text { at level } r, \\
0, & \text { otherwise }\end{cases} \\
& p_{i j r}=\text { conditional probability that facility } j \text { serves customer } i \text { at level } r, \\
& \text { given that } i \text { is assigned to } j \text { at level } r
\end{aligned}
$$

Given these definitions, $p_{i j r} z_{i j r}$ is the probability (i.e., the expected fraction of time) that facility $j$ serves customer $i$ at level $r$. The formulation enforces the relationships among these decision values to ensure that $p_{i j r}$ accounts for customer $i$ 's other assigned facilities at levels 0 through $r-1$.

\subsection{Formulation}

\subsubsection{Inventory Cost Expression}

In this section, we derive an exact formulation for the expected inventory cost as a function of the decision variables. The expected inventory cost function includes both ordering and holding costs. Like 
the LMRP, the HRLMRP assumes that each DC follows a $(Q, R)$ policy with a service level constraint (which applies when the DC is operational). This policy decomposes into a cycle-stock component (which comes from the Economic Order Quantity (EOQ) model) and a safety-stock component (to protect against demand uncertainty). More importantly, the optimal expected cost of such a policy can be expressed in closed form (see, e.g., Snyder and Shen 2011). If there were no disruptions, then using our notation, the optimal expected inventory cost at facility $j$ is given by

$$
K_{j} \sqrt{\sum_{i} \mu_{i}}
$$

where the sum is over all customers that are served by facility $j$, and

$$
K_{j}=\sqrt{2 \theta h_{j}\left(F_{j}+\beta g_{j}\right)}+\theta h_{j} z_{\alpha} \sqrt{L \gamma}
$$

(Recall that $\mu_{i}$ is the mean demand of customer $i$, as well as its variance divided by $\gamma$.) In the presence of disruptions, (1) gives the expected inventory cost rate in a given failure state, assuming we reinterpret the sum to include all customers that are served by $j$ in that state.

Note that when a disruption begins or ends and the customers are reassigned, a given facility's inventory policy does not change until its next replenishment cycle. Therefore, during a portion of some replenishment cycles, the inventory systems are not operating optimally because they do not yet reflect the new customer assignments. To avoid this complication, we will assume that disruptions begin and end relatively slowly compared to the inventory cycles, so that it is reasonable to ignore these transitional intervals.

The expected inventory cost can be separated based on how many DCs fail.

1) If no DCs fail, then from (1), the expected inventory cost is

$$
C_{0}=\prod_{j \in J}\left(1-q_{j}\right)^{y_{j}} \cdot \sum_{j \in J} K_{j} \sqrt{\sum_{i \in I} \mu_{i} z_{i j 0}}
$$

since $i$ is assigned to $j$ if and only if $z_{i j 0}=1$, and since $j$ fails with probability $q_{j}$.

2) If only one DC $k$ fails, then customer $i$ will be assigned to a given facility $j$ if $j$ has not failed and if either $z_{i j 0}=1$ or $z_{i k 0}=z_{i j 1}=1$. Therefore, the expected inventory cost is

$$
C_{1}=\sum_{k \in J}\left[q_{k} y_{k} \prod_{j \in J \backslash k}\left(1-q_{j}\right)^{y_{j}} \cdot \sum_{j \in J \backslash k} K_{j} \sqrt{\sum_{i \in I} \mu_{i} z_{i j 0}+\sum_{i: z_{i k 0}=1} \mu_{i} z_{i j 1}}\right] .
$$

3) If two DCs $k$ and $l$ fail, then $i$ will be assigned to $j$ if $j$ has not failed, and if $z_{i j 0}=1$ or $z_{i k 0}+z_{i l 0}=z_{i j 1}=1$ or $z_{i k 0}+z_{i l 0}=z_{i k 1}+z_{i l 1}=z_{i j 2}=1$. Let $I_{0}=I ; I_{1}=\left\{i \in I \mid z_{i k 0}+z_{i l 0}=1\right\} ;$ 
and $I_{2}=\left\{i \in I \mid z_{i k 0}+z_{i l 0}=z_{i k 1}+z_{i l 1}=1\right\}$. Then the expected inventory cost when two DCs fails is

$$
C_{2}=\sum_{k, l \in J}\left[q_{k} y_{k} q_{l} y_{l} \prod_{j \in J \backslash\{k, l\}}\left(1-q_{j}\right)^{y_{j}} \cdot \sum_{j \in J \backslash\{k, l\}} K_{j} \sqrt{\sum_{r=0}^{2} \sum_{i \in I_{r}} \mu_{i} z_{i j r}}\right] .
$$

In general, suppose that $n$ DCs fail. Let $J^{\prime} \subseteq J$ be the set of failed DCs $\left(\left|J^{\prime}\right|=n\right)$. Let $I_{0}=I$ and

$$
I_{r}=\left\{i \in I \mid \sum_{k \in J^{\prime}} z_{i k s}=1, s=0,1, \cdots, r-1\right\} .
$$

Then the expected inventory cost when $n$ DCs fail is

$$
C_{n}=\sum_{J^{\prime} \subseteq J:\left|J^{\prime}\right|=n}\left[\prod_{k \in J^{\prime}} q_{k} y_{k} \cdot \prod_{j \in J \backslash J^{\prime}}\left(1-q_{j}\right)^{y_{j}} \cdot \sum_{j \in J \backslash J^{\prime}} K_{j} \sqrt{\sum_{r=0}^{n} \sum_{i \in I_{r}} \mu_{i} z_{i j r}}\right] .
$$

We now have the following result:

Proposition 1 The expected inventory cost is given by

$$
C=\sum_{n=0}^{|J|-1} \sum_{J^{\prime} \subseteq J:\left|J^{\prime}\right|=n}\left[\prod_{k \in J^{\prime}} q_{k} y_{k} \cdot \prod_{j \in J \backslash J^{\prime}}\left(1-q_{j}\right)^{y_{j}} \cdot \sum_{j \in J \backslash J^{\prime}} K_{j} \sqrt{\sum_{r=0}^{n} \sum_{i \in I_{r}} \mu_{i} z_{i j r}}\right]
$$

The expression in (3) is difficult to embed into the objective function of an optimization problem because of the combinatorial expression $\sum_{J^{\prime} \subseteq J}$ and the fact that the set $I_{r}$ depends on the values of the decision variables $\boldsymbol{z}$, and the nonlinearity depends on $\boldsymbol{y}$. Therefore, we propose to approximate the expected cost using the following expression:

$$
C^{\prime}=\sum_{j \in J} K_{j} \sqrt{\sum_{r=0}^{R-1} \sum_{i \in I} \mu_{i} p_{i j r} z_{i j r}}=\sum_{j \in J} \sqrt{\sum_{i \in I} \sum_{r=0}^{R-1} \hat{K}_{i j} p_{i j r} z_{i j r}}
$$

where $\hat{K}_{i j}=\mu_{i} K_{j}^{2}$.

The essence of this approximation is to move the expectation over the failure states to the inside of the square-root term; that is, to replace (1) the expectation (over the failure states) of the inventory cost of the assigned demand by (2) the inventory cost of the expectation (over the failure states) of the assigned demand. In particular, since $p_{i j r} z_{i j r}$ is the probability that facility $j$ serves customer $i$ at level $r$, the expected demand assigned to $j$ is $D_{j}=\sum_{i \in I} \sum_{r=0}^{R-1} \mu_{i} p_{i j r} z_{i j r}$. Put another way, we are replacing $\mathbb{E}_{S}\left[G_{j}\left(I_{j}(S)\right)\right]$ with $G_{j}\left(\mathbb{E}_{S}\left[I_{j}(S)\right]\right)$, where $I_{j}(S)$ is the set of customers served by $j$ in a given failure state $S, G_{j}\left(I_{j}\right)$ is the expected inventory cost at $j$ given that $I_{j}$ is the set of customers served by $j$, and $\mathbb{E}_{S}[\cdot]$ denotes expectation with respect to the failure states. Therefore, 
we use $C^{\prime}=\sum_{j \in J} G_{j}\left(\mathbb{E}_{S}\left[I_{j}(S)\right]\right)=\sum_{j \in J} K_{j} \sqrt{D_{j}}$, which has a similar form as the inventory cost in Daskin et al. (2002). We demonstrate numerically in 86.1 that our approximation is quite accurate, even when the $q_{j}$ are facility-dependent.

Note that if $q_{j}=q$ for all $j \in J$, then $C^{\prime}$ is equivalent to the inventory cost used by Chen et al. (2011) in their LMRP model with disruptions. They obtain their expression by replacing the demand served by a facility with its mean, though they do not provide further justification.

\subsubsection{Formulation}

In addition to the inventory cost formulated in 3.2 .1 , the objective function of the HRLMRP has two other terms, corresponding to the fixed cost of opening facilities and the expected transportation cost. The fixed cost is the same as in the UFLP: $\sum_{j \in J} f_{j} y_{j}$. The expected transportation cost is

$$
\beta \sum_{i \in I} \sum_{j \in J_{e}} \sum_{r=0}^{R} \mu_{i} c_{i j} p_{i j r} z_{i j r}+\beta \sum_{j \in J_{e}} D_{j} a_{j}=\sum_{i \in I} \sum_{j \in J_{e}} \sum_{r=0}^{R} \hat{c}_{i j} p_{i j r} z_{i j r},
$$

where $\hat{c}_{i j}=\beta \mu_{i}\left(c_{i j}+a_{j}\right)$. The heterogeneous reliable location model with risk pooling (HRLMRP) can now be formulated as follows:

$$
\min \sum_{j \in J} f_{j} y_{j}+\sum_{i \in I} \sum_{j \in J} \sum_{r=0}^{R} \hat{c}_{i j} p_{i j r} z_{i j r}+\sum_{j \in J} \sqrt{\sum_{i \in I} \sum_{r=0}^{R-1} \hat{K}_{i j} p_{i j r} z_{i j r}}
$$

s.t.

$$
\begin{aligned}
& \sum_{j \in J} z_{i j r}+\sum_{s=0}^{r} z_{i e s}=1 \quad \forall i \in I, 0 \leq r \leq R \\
& z_{i j r} \leq y_{j} \quad \forall i \in I, j \in J, 0 \leq r \leq R-1 \\
& \sum_{r=0}^{R-1} z_{i j r} \leq 1 \quad \forall i \in I, j \in J \\
& \sum_{r=0}^{R} z_{i e r}=1 \quad \forall i \in I \\
& p_{i j 0}=1-q_{j} \quad \forall i \in I, j \in J_{e} \\
& p_{i j r}=\left(1-q_{j}\right) \sum_{k \in J} \frac{q_{k}}{1-q_{k}} p_{i, k, r-1} z_{i, k, r-1} \quad \forall i \in I, j \in J_{e}, 1 \leq r \leq R \\
& y_{j}, z_{i j r} \in\{0,1\} \quad \forall i \in I, j \in J_{e}, 0 \leq r \leq R
\end{aligned}
$$

The objective function (6) is the sum of the facility setup costs, expected transportation costs, and expected inventory costs. Constraints (7) enforce that for each customer $i$ and each level $r$, either $i$ is 
assigned to a regular facility at level $r$ or it is assigned to the dummy facility $e$ at some level $s \leq r$. Constraints (8) ensure that customers are only assigned to the open facilities, and constraints (9) guarantee that each customer $i$ is not assigned to a given facility $j$ at more than one level. Constraints (10) require each customer to be assigned to the dummy facility at some level, to capture the possibility that all open facilities fail and the customer incurs the lost sales cost. Constraints (11) and (12) are the "transitional probability" equations. Note that $p_{i j 0} z_{i j 0}$, the probability that facility $j$ serves customer $i$ at level 0 , is simply the probability that $j$ remains open, so $p_{i j 0}=1-q_{j}$; for $1 \leq r \leq R, p_{i j r}$ is equal to $\left(1-q_{j}\right) q_{k} p_{i, k, r-1} /\left(1-q_{k}\right)$ given that facility $k$ serves customer $i$ at level $r-1$. This approach is due to Cui et al. (2010), and we refer the reader to that reference or to Aboolian et al. (2012) for details. Finally, constraints 13 are integrality constraints.

\subsection{Remarks}

Like the RLMRP with homogeneous failure probabilities (Chen et al. 2011), the HRLMRP seeks to balance the transportation cost (i.e., by spreading out customer demand across a large number of facilities) and the inventory and the facility setup costs (i.e., by pooling demand at a few facilities).

The HRLMRP has several important special cases. If $q_{j}=q$ for all $j \in J$, then the probability that a customer receives service from its level- $r$ facility is $q^{r-1}(1-q)$. Thus the decision variables $p_{i j r}=q^{r-1}(1-q)$ are constant for different $i$ and $j$. In this case, the proposed formulation is identical to the RLMRP models (with homogeneous failure probabilities) in Jeon (2008), Aryanezhad et al. (2010) and Chen et al. (2011).

If, instead, customers face deterministic demands and we ignore the inventory cost and the orders from facilities to the supplier (in other words, if we set $g_{j}=0, a_{j}=0, F_{j}=0, h_{j}=0, L=0, \beta=$ $1, \theta=0$ for all $j \in J)$, then we obtain the RFLP with heterogeneous failure probabilities formulated by Cui et al. (2010) and Aboolian et al. (2012).

Moreover, if all of the facilities are prefectly reliable, i.e., $q_{j}=0 \forall j \in J$, then all assignments are at the primary level, i.e., $R=1$ and the HRLMRP reduces to the LMRP proposed by Daskin et al. (2002) and Shen et al. (2003).

Finally, if all facilities are perfectly reliable, we consider only the primary assignment, and we ignore the inventory costs (by setting $g_{j}=a_{j}=F_{j}=h_{j}=q_{j}=0, R=1$ ), then the model is just the classical UFLP.

Obviously, the HRLMRP is NP-hard because the UFLP is a special case, and therefore no known exact methods can solve the HRLMRP in polynomial time. Moreover, the multiplicative term $p_{i j r} z_{i j r}$, 
the square-root term and the scaling that exist in the objective function make this model very challenging to solve. The additional incorporation of a large number of probabilistic failure scenarios further complicates the problem. In the following two sections, we will first propose an approach using SOS2 that can find the exact solution for small-scale instances, and then design a customized Lagrangian relaxation heuristic that can find a good solution for larger instances efficiently.

\section{SOS2 Reformulation}

Formulation (HRLMRP) is nonlinear due to both the square root terms in the objective and the multiplicative terms $p_{i j r} z_{i j r}$, each a product of a continuous variable and a binary variable. We address the former nonlinearity using SOS2 and the latter using the McCormick inequalities (McCormick 1976), also known as the reformulation-linearization technique (RLT; Sherali and Alameddine 1992); see also Luedtke et al. (2012).

\subsection{Linearization of the Multiplicative Terms}

As in Cui et al. (2010), we use the McCormick/RLT inequalities, replacing each $p_{i j r} z_{i j r}$ with a new variable $w_{i j r}$. For each $i \in I, j \in J, 0 \leq r \leq R$, a new set of constraints is added to the formulation to enforce $w_{i j r}=p_{i j r} z_{i j r}$ :

$$
\begin{aligned}
& w_{i j r} \leq p_{i j r} \\
& w_{i j r} \leq z_{i j r} \\
& w_{i j r} \geq 0 \\
& w_{i j r} \geq p_{i j r}+z_{i j r}-1
\end{aligned}
$$

Now, the resulting formulation (HRLMRP-L) is stated below:

$$
\min \sum_{j \in J} f_{j} y_{j}+\sum_{i \in I} \sum_{j \in J_{e}} \sum_{r=0}^{R} \hat{c}_{i j} w_{i j r}+\sum_{j \in J_{e}} \sqrt{\sum_{r=0}^{R} \sum_{i \in I} \hat{K}_{i j} w_{i j r}}
$$

s.t.

(7)-(11), 13)- 17

$$
p_{i j r}=\left(1-q_{j}\right) \sum_{k \in J} \frac{q_{k}}{1-q_{k}} w_{i, k, r-1} \quad \forall i \in I, j \in J, 1 \leq r \leq R
$$




\subsection{Linearization of the Square Root Terms}

Special ordered sets of type 2 (SOS2) are a set of consecutive variables in which not more than two adjacent members may be non-zero in a feasible solution. The concave terms in the (HRLMRP-L) objective function (18) can be approximated by linear terms using SOS2. There are $|J|$ square root functions. Let

- $V=$ set of breakpoints, indexed by $v$;

- $\rho_{v j}=v$ th SOS2 variable for the square root function for facility $j$;

- $T_{v j}=v$ th breakpoint of the square root function for facility $j$;

- $t_{j}=$ any point in the closed interval $\left[T_{1 j}, T_{|V| j}\right]$ in the square root function for facility $j$.

A point $t_{j}$ can be expressed as a linear combination of the breakpoints. Also, $\sqrt{t_{j}}$ can be approximated by a linear combination of the square root values of the breakpoints. So for all $j \in J$, we have

$$
\begin{aligned}
& \sqrt{\sum_{r=0}^{R} \sum_{i \in I} \hat{K}_{i j} w_{i j r}}=\sqrt{t_{j}} \geq \sqrt{T_{1 j}} \rho_{1 j}+\cdots+\sqrt{T_{|V| j}} \rho_{|V| j}=\sum_{v \in V} \sqrt{T_{v j}} \rho_{v j} \\
& \sum_{r=0}^{R} \sum_{i \in I} \hat{K}_{i j} w_{i j r}=t_{j}=T_{1 j} \rho_{1 j}+\cdots+T_{|V| j} \rho_{|V| j}=\sum_{v \in V} T_{v j} \rho_{v j} \\
& \rho_{1 j}+\cdots+\rho_{|V| j}=\sum_{v \in V} \rho_{v j}=1 \\
& \rho_{v j} \geq 0 \quad \forall v \in V
\end{aligned}
$$

That is, 20 can approximate the concave terms in the objective function when subject to constraints 21 - 23). Then, the approximate formulation (HRLMRP-SOS2) is

$$
\min \sum_{j \in J} f_{j} y_{j}+\sum_{i \in I} \sum_{j \in J_{e}} \sum_{r=0}^{R} \hat{c}_{i j} w_{i j r}+\sum_{j \in J_{e}} \sum_{v \in V} \sqrt{T_{v j}} \rho_{v j}
$$

s.t.

(7)-(11), (13)-(17), (19), (21)-(23),

where $\rho_{v j}, \forall v \in V, j \in J$ are the SOS2 variables.

Formulation (HRLMRP-SOS2) is linear and can solved using an off-the-shelf MIP solver such as CPLEX or Gurobi in a straightforward way. 


\section{The Lagrangian Relaxation Algorithm}

\subsection{Formulation}

The difficulty of solving (HRLMRP-SOS2) exactly for large instances motivates the development of a Lagrangian relaxation (LR) heuristic. Relaxing constraints (7) in (HRLMRP) with multipliers $\boldsymbol{\lambda}$ yields a Lagrangian subproblem (HRLMRP-LR) with the following objective function:

$$
\begin{aligned}
\phi(\lambda) & =\min \sum_{j \in J} f_{j} y_{j}+\sum_{i \in I} \sum_{j \in J} \sum_{r=0}^{R} \hat{c}_{i j} p_{i j r} z_{i j r}+\sum_{j \in J} \sqrt{\sum_{i \in I} \sum_{r=0}^{R-1} \hat{K}_{i j} p_{i j r} z_{i j r}}+\sum_{i \in I} \sum_{r=0}^{R} \lambda_{i r}\left(1-\sum_{j \in J} z_{i j r}-\sum_{s=0}^{r} z_{i e s}\right) \\
& =\min \sum_{j \in J} f_{j} y_{j}+\sum_{j \in J_{e}} \sum_{i \in I} \sum_{r=0}^{R} \varphi_{i j r} z_{i j r}+\sum_{j \in J} \sqrt{\sum_{i \in I} \sum_{r=0}^{R-1} \xi_{i j r} z_{i j r}+\sum_{i \in I} \sum_{r=0}^{R} \lambda_{i r},}
\end{aligned}
$$

where

$$
\begin{gathered}
\varphi_{i j r}= \begin{cases}\hat{c}_{i j} p_{i j r}-\lambda_{i r}, & \text { if } j \in J \\
\hat{c}_{i e} p_{i e r}-\sum_{s=r}^{R} \lambda_{i s}, & \text { if } j=e\end{cases} \\
\xi_{i j r}= \begin{cases}\hat{K}_{i j} p_{i j r}, & \text { if } j \in J \\
0, & \text { if } j=e .\end{cases}
\end{gathered}
$$

The equality follows from a similar algebraic manipulation as in Snyder and Daskin (2005).

The relaxed problem can be decomposed into a set of subproblems, one for each $j \in J_{e}$. In particular,

for each $j$, we need to decide whether to set $y_{j}$ to 0 or 1 , and then how to set the $z_{i j r}$ variables optimally. Obviously, if $y_{j}=0$, then $z_{i j r}=0$ for all $i \in I, 0 \leq r \leq R-1$. The more interesting case is when $y_{j}=1$. For each $j \in J$, we have to solve the resulting relaxed problem:

$$
\left(\mathrm{RP}_{j}\right) \quad \bar{\phi}_{j}(\boldsymbol{\lambda})=\min _{z} \sum_{i \in I} \sum_{r=0}^{R} \varphi_{i r} z_{i r}+\sqrt{\sum_{i \in I} \sum_{r=0}^{R} \xi_{i r} z_{i r}}
$$

s.t.

$$
\begin{aligned}
& \sum_{r=0}^{R} z_{i r}=1 \quad \forall i \in I \\
& z_{i r} \in\{0,1\} \quad \forall i \in I, 0 \leq r \leq R
\end{aligned}
$$


For notational simplicity, we omit subscript $j$ in all relevant variables and parameters. We also introduce binary variables $z_{i R}$, which represent not assigning $i$ to $j$ at any level, and therefore act as slack variables, allowing us to replace the inequality (9) with the equality (29). In addition, we omit the constant term $f_{j}$, and further define $\varphi_{i R}=0$ and $\xi_{i R}=0$ for all $i \in I$. Therefore, if $j=e$, the objective function simplifies to

$$
\bar{\phi}_{e}(\boldsymbol{\lambda})=\min _{\boldsymbol{z}} \sum_{i \in I} \sum_{r=0}^{R} \varphi_{i r} z_{i r} .
$$

The quantity $\bar{\phi}_{j}$ is called the benefit of opening facility $j$.

\subsection{Lower Bound}

\subsubsection{A Lower Bound on $p_{i j r}$.}

Note that in (25), the coefficients $\varphi_{i j r}$ and $\xi_{i j r}$ both depend on $p_{i j r}$, which is a decision variable. Let $q_{[0]} \leq q_{[1]} \leq \cdots \leq q_{[|J|-1]}$ be an ordering of the failure probabilities of the facilities in $J$. For $j \in J_{e}$, define

$$
Q_{j r}=\left(\prod_{t=0}^{r-1} q_{[t]}\right)\left(1-q_{j}\right) .
$$

Aboolian et al. (2012) prove that $Q_{j r} \leq p_{i j r}$. The basic idea is to assume that the facilities assigned to $i$ at the $r$ lowest assignment levels are the $r$ facilities with the smallest failure probability. Aboolian et al. (2012) further show that replacing $p_{i j r}$ with $Q_{j r}$ in their formulation (for the RFLP with heterogenous failure probabilities) leads to a lower bound on the optimal objective function value. However, this bound may be weak. Therefore, we propose the following tighter lower bound on $p_{i j r}$.

Let $Q_{j r}(\mathcal{J})$ be as defined in 32 but with the $q_{[t]}$ redefined to include only those facilities in $\mathcal{J}$, for a fixed subset $\mathcal{J} \subseteq J_{e}$. Then we have the following:

Lemma 1 If $\mathcal{J}_{1} \subseteq \mathcal{J}_{2} \subseteq J_{e}$, then $p_{i j r} \geq Q_{j r}\left(\mathcal{J}_{1}\right) \geq Q_{j r}\left(\mathcal{J}_{2}\right) \geq Q_{j r}\left(J_{e}\right)$.

Proof. The proof is provided in Appendix C.1.

We now propose a modification to the lower bound by Aboolian et al. (2012) that exploits Lemma 1. For each customer $i \in I$, we would like to exclude from $\mathcal{J}$ those facilities that will for sure not be assigned to $i$ in the optimal solution. First, we eliminate those facilities that are forced out of the solution using the variable fixing technique $(\$ 5.6)$ or fixed to 0 in the branch and bound ( $\$ 5.7$ process. Second, we can eliminate certain facilities from $\mathcal{J}$ when evaluating $Q_{j r}(\mathcal{J})$ for a given customer $i$, based on $i$ 's "preference" among the facilities (in a sense to be defined shortly). 
The idea is as follows. For our problem, as in the LMRP, it is sometimes optimal for a customer to be assigned to a facility that is not its closest open facility, as a result of the inventory costs' concave dependence on the demand assigned. However, it is sometimes possible to establish that for a given customer $i$, it will always be cheaper to assign $i$ to a certain facility $j$ than to another facility $k$, no matter how much demand is assigned to each. If this is the case, we know that if $j$ is open, then $i$ will be assigned to $j$ at a lower level than to $k$. Moreover, if the number of such "preferred" open facilities is greater than $r$, then we will never assign $i$ to $k$ at level $r$ since there are $r$ better facilities available. Thus, in this case we can eliminate $k$ from $\mathcal{J}$ when evaluating $Q_{j r}(\mathcal{J})$ for $i$.

Proposition 2 Suppose that, for some $i \in I, j, k \in J_{e}$ such that $y_{j}=y_{k}=1$, the following inequality holds:

$$
\hat{c}_{i j}+\sqrt{\hat{K}_{i j}}<\hat{c}_{i k}-\sqrt{\sum_{i^{\prime} \in I} \hat{K}_{i^{\prime} k}}+\sqrt{\sum_{i^{\prime} \in I} \hat{K}_{i^{\prime} k}+\hat{K}_{i k}} .
$$

Then it is not optimal to assign $i$ to $k$ at a lower level than $j$; that is, it is not optimal to set $z_{i, k, r_{1}}=$ $z_{i, j, r_{2}}=1$ for $r_{2}>r_{1}$ in the solution to (HRLMRP).

Proof. The theoretical proof is provided in Appendix C.2.

In fact, we believe that a stronger inequality is true of all optimal solutions:

Conjecture 1 Inequality (33) can be replaced by the following inequality in Proposition 2:

$$
\hat{c}_{i j}+\sqrt{\hat{K}_{i j}}<\hat{c}_{i k}-\sqrt{\sum_{i^{\prime} \in I} \hat{K}_{i^{\prime} k}-\hat{K}_{i k}}+\sqrt{\sum_{i^{\prime} \in I} \hat{K}_{i^{\prime} k}} .
$$

We have not been able to prove this inequality algebraically. However, we conducted an extensive computational experiment and found that, for every instance we tested, Conjecture 1 holds. (See Appendix B.) Therefore, in what follows, we use (34) to identify assignment patterns that cannot be optimal. (On the other hand, we also note that the numerical test shows that the weaker inequality (33) is already very tight in most cases.)

Let $\hat{r}_{i k}$ be the number of facilities $j$ that have been forced into the solution and for which (34) holds, i.e., the number of facilities that are definitely "preferable" to $k$ for customer $i$. (A facility $j$ is considered "forced in" if $y_{j}$ is fixed to 1 by the branch-and-bound process or the variable-fixing method.) Then for each level $0 \leq r \leq R$, if $\hat{r}_{i k}>r$, we eliminate $k$ from $\mathcal{J}$ when evaluating $Q_{j r}(\mathcal{J})$ for $i$.

To summarize, for all $i \in I, j, k \in J_{e}, p_{i j r} \leq Q_{j r}\left(\mathcal{J}_{i}\right)$, where $\mathcal{J}_{i}$ consists of $J_{e}$ minus all facilities $k$ such that either: 
- $k$ is forced out of the solution by the variable-fixing or branch-and-bound processes, or

- $\hat{r}_{i k}>r$.

We propose to replace $p_{i j r}$ with $Q_{j r}\left(\mathcal{J}_{i}\right)$ in $\left(\mathrm{RP}_{j}\right)$. We call the resulting problem $\left(\mathrm{RP}_{j}^{\prime}\right)$, and the overall Lagrangian subproblem (HRLMRP-LR'). In $\$ 5.2 .2$, we show how to solve $\left(\mathrm{RP}_{j}^{\prime}\right)$.

Lemma 2 The optimal objective value of $\left(R P_{j}^{\prime}\right)$ is a lower bound on that of the original $\left(R P_{j}\right)$, and therefore on that of (HRLMRP).

Proof. See Appendix C.3.

For most facility location problems, the lower bound from Lagrangian relaxation may be strictly less than the optimal objective value for the original problem (i.e., there may be an optimality gap), but this gap can be closed by branching on the location variables. Unfortunately, this is not true for our problem. Under some conditions, $Q_{j r}\left(\mathcal{J}_{i}\right)$ will be strictly less than $p_{i j r}$. When this happens, the optimality gap from the Lagrangian relaxation will be strictly positive, even after branching on the $\boldsymbol{y}$ variables. The only way to ensure that the optimality gap goes to 0 is to branch on the $\boldsymbol{z}$ variables as well, which is impractical. This is why we treat our Lagrangian relaxation approach as a heuristic rather than an exact algorithm.

If any of the following conditions is violated, then $Q_{j r}\left(\mathcal{J}_{i}\right)$ may be strictly less than $p_{i j r}$ :

1. All non-open facilities are forced out of the solution by the variable-fixing or branch-and-bound process.

2. For a given customer $i$, all facilities to which $i$ is not assigned at any level are forced out of the solution by the variable-fixing or branch-and-bound process.

3. Each customer is assigned to facilities in order of their failure probabilities, i.e., $z_{i j r}=z_{i, k, r+1}=1$ only if $q_{j} \leq q_{k}$.

To see this, suppose $j$ is not forced out of the solution; then it will be included in $\mathcal{J}_{i}$, so it will be included in the product for $Q_{j r}\left(\mathcal{J}_{i}\right)$ in $(32)$. On the other hand, if $y_{j}=0$ (condition 1) or if $z_{i j r}=0$ for all $r$ (condition 2), then $j$ will not be included in the product for $p_{i j r}$ in (C1). Furthermore, $Q_{j r}(\mathcal{J})$ is calculated by first sorting the facilities in increasing order of failure probability, so if $i$ 's lower-level facility has a larger failure probability than a higher-level facility, $Q_{j r}(\mathcal{J})$ may be less than $p_{i j r}$. Unfortunately, these conditions are violated frequently. Still, $Q_{j r}(\mathcal{J})$ is a useful estimate of $p_{i j r}$. 


\subsubsection{Solving the Relaxed Subproblem.}

In this section, we will propose an efficient method to solve the modified Lagrangian subproblem $\left(\mathrm{RP}_{j}^{\prime}\right)$ (the subproblem $\left(\mathrm{RP}_{j}\right)$ with $p_{i j r}$ replaced by $Q_{j r}\left(\mathcal{J}_{i}\right)$ ).

For $j=e$, the objective function of $\left(\mathrm{RP}_{j}^{\prime}\right)$ reduces to (31), which is linear. This problem is easy to solve: For each customer $i \in I$, we simply set $z_{i r_{0}}=1$ for $r_{0}=\underset{r=0, \cdots, R}{\arg \min } \varphi_{i r}$.

Now suppose $j \neq e$. If constraint 29 were omitted, $\left(\mathrm{RP}_{j}^{\prime}\right)$ would decouple by $r$ into $R$ separate problems, each of which is equivalent to the subproblem introduced by Daskin et al. (2002) and Shen et al. (2003), for which they propose an efficient solution algorithm with complexity $O(|I| \log |I|)$. However, constraint (29) prohibits this decoupling, resulting in an objective function with multiple concave terms and rendering the method by Daskin et al. (2002) and Shen et al. (2003) inapplicable. However, our subproblem $\left(\mathrm{RP}_{j}^{\prime}\right)$ is equivalent to the subproblem faced by Chen et al. (2011), who propose a customized polynomial-time algorithm to solve it. We briefly summarize their method and contribute some modifications that improve its performance.

From this point forward, we assume that $\varphi_{i j r}$ and $\xi_{i j r}$ have been modified by replacing the variable $p_{i j r}$ with the parameter $Q_{j r}\left(\mathcal{J}_{i}\right)$ in $(26)$ and $(27)$, and we continue to omit the subscript $j$ when referring to these parameters in the context of the problems $\left(\mathrm{RP}_{j}\right)$ and $\left(\mathrm{RP}_{j}^{\prime}\right)$.

Suppose we have a partial solution $z$ in which, for some $i \in I, z_{i r}$ has not yet been set to 1 for any $r$. Chen et al. (2011) define the marginal contribution of setting $z_{i r}=1$ as $M_{i r}\left(w_{i}\right)=\sqrt{w_{i}+\xi_{i r}}-\sqrt{w_{i}}+\varphi_{i r}$, for $i \in I, r \in\{0,1, \cdots, R\}$, where

$$
w_{i}=\sum_{k \in I \backslash\{i\}} \sum_{r=0}^{R} \xi_{k r} z_{k r} .
$$

Note that $M_{i r}(0)=\sqrt{\xi_{i r}}+\varphi_{i r}$. Chen et al. (2011) define $R_{i}$ as a maximal subset of $\{0,1, \cdots, R\}$

whose elements have distinct $\left(\xi_{i r}, \varphi_{i r}\right)$ pairs, i.e., a maximal subset such that $\left(\xi_{i r}, \varphi_{i r}\right) \neq\left(\xi_{i r^{\prime}}, \varphi_{i r^{\prime}}\right)$ if $r \neq r^{\prime} \in R_{i}$. They further define

$$
N_{i}=\left\{r \in R_{i} \mid \text { for all } r^{\prime} \in R_{i} \backslash\{r\} \text {, either } M_{i r}(0)<M_{i r^{\prime}}(0) \text { or } \varphi_{i r}<\varphi_{i r^{\prime}}\right\},
$$

i.e., each element $r \in N_{i}$ must satisfy one of the following three conditions: $(1) M_{i r}(0)$ is the minimum; (2) $\varphi_{i r}$ is the minimum; (3) either $M_{i r}(0)$ or $\varphi_{i r}$ is smaller than any other element of $N_{i}$.

Chen et al. (2011) assert that the continuous functions $M_{i r}\left(w_{i}\right)$ and $M_{i r^{\prime}}\left(w_{i}\right)$ intersect at

$$
\bar{w}_{r r^{\prime}}^{i}=\frac{\left(\xi_{i r}-\xi_{i r^{\prime}}\right)^{2}}{4\left(\varphi_{i r}-\varphi_{i r^{\prime}}\right)^{2}}+\frac{\left(\varphi_{i r}-\varphi_{i r^{\prime}}\right)^{2}}{4}-\frac{\xi_{i r}+\xi_{i r^{\prime}}}{2}>0 .
$$


(We provide a proof of an equivalent expression in Appendix C.4.) From this expression, Chen et al. (2011) define a sequence of intervals in $[0, \infty)$ as follows. First, they sort the elements of $N_{i}$ into an ordered sequence $r(i, 0), r(i, 1), \cdots, r\left(i,\left|N_{i}\right|-1\right)$ such that $M_{i, r(i, k)}(0)<M_{i, r(i, k+1)}(0)$ and $\varphi_{i, r(i, k)}>$ $\varphi_{i, r(i, k+1)}$. (Note that these ensure that $\left.\xi_{i, r(i, k)}<\xi_{i, r(i, k+1)}.\right)$ Then, they define

$$
\begin{aligned}
& w_{k}^{i-}= \begin{cases}0, & k=0 \\
\max _{k^{\prime}=0, \cdots, k-1} \bar{w}_{r(i, k), r\left(i, k^{\prime}\right)}^{i}, & k=1, \cdots,\left|N_{i}\right|-1\end{cases} \\
& w_{k}^{i+}= \begin{cases}\min _{k^{\prime}=k+1, \cdots,\left|N_{i}\right|-1} \bar{w}_{r(i, k), r\left(i, k^{\prime}\right)}^{i}, & k=0, \cdots,\left|N_{i}\right|-2 \\
\infty, & k=\left|N_{i}\right|-1\end{cases}
\end{aligned}
$$

Chen et al. (2011) claim that the intervals $\left[w_{k}^{i-}, w_{k}^{i+}\right], k=0, \cdots,\left|N_{i}\right|-1$, form a non-overlapping partition of $[0, \infty)$ from left to right.

However, we find that this definition does not guarantee a non-overlapping partition of $[0, \infty)$ in some situations. Appendix $\mathrm{D}$ provides a counterexample. We now propose some improvements to the definitions above. We define

$$
\mathcal{F}_{k j}^{i}=\varphi_{i, r(i, k)}+\frac{\xi_{i, r(i, k)}-\xi_{i, r(i, j)}}{\varphi_{i, r(i, j)}-\varphi_{i, r(i, k)}} .
$$

We first show that the following proposition holds:

Proposition 3 If the elements of $N_{i}$ are sorted into an ordered sequence $r(i, 0), r(i, 1), \cdots, r\left(i,\left|N_{i}\right|-1\right)$ such that $M_{i, r(i, k)}(0)<M_{i, r(i, k+1)}(0)$ and $\varphi_{i, r(i, k)}>\varphi_{i, r(i, k+1)}$ for all $k=0,1, \cdots,\left|N_{i}\right|-2$, then for any three elements $r(i, j), r(i, k), r(i, s) \in N_{i}$ such that $j<k<s$ :

(1) If $\mathcal{F}_{j k}^{i} \geq \mathcal{F}_{s k}^{i}$, then $w_{r(i, k)}^{i-} \geq w_{r(i, k)}^{i+}$ (or the interval $\left[w_{r(i, k)}^{i-}, w_{r(i, k)}^{i+}\right]$ is empty).

(2) If $\mathcal{F}_{j k}^{i}<\mathcal{F}_{s k}^{i}$, then $\bar{w}_{r(i, j), r(i, k)}^{i}<\bar{w}_{r(i, j), r(i, s)}^{i}<\bar{w}_{r(i, k), r(i, s)}^{i}$.

Proof. (1) From Lemma 4 in Appendix C.4. if $\mathcal{F}_{j k}^{i} \geq \mathcal{F}_{s k}^{i}$, then $\bar{w}_{r(i, j), r(i, k)}^{i} \geq \bar{w}_{r(i, k), r(i, s)}^{i}$. From the definition by Chen et al. (2011),

$$
\begin{aligned}
& w_{r(i, k)}^{i-}=\max _{k^{\prime}=0, \cdots, k-1} \bar{w}_{\left(r\left(i, k^{\prime}\right), r(i, k)\right.}^{i} \geq \bar{w}_{r(i, j), r(i, k)}^{i} \\
& w_{r(i, k)}^{i+}=\min _{k^{\prime}=k+1, \cdots,|N|-1} \bar{w}_{r(i, k), r\left(i, k^{\prime}\right)}^{i} \leq \bar{w}_{r(i, k), r(i, s)}^{i} .
\end{aligned}
$$

Consequently, $w_{r(i, k)}^{i-} \geq w_{r(i, k)}^{i+}$.

(2) From Lemma 4 in Appendix C.4. if $\mathcal{F}_{j k}^{i}<\mathcal{F}_{s k}^{i}$, then the intersections satisfy $\bar{w}_{r(i, j), r(i, k)}^{i}<$ $\bar{w}_{r(i, j), r(i, s)}^{i}<\bar{w}_{r(i, k), r(i, s)}^{i}$. 
After sorting the elements in $N_{i}$, for any three elements $r(i, j), r(i, k), r(i, s) \in N_{i}$ such that $j<k<$ $s$, if $\mathcal{F}_{j k}^{i} \geq \mathcal{F}_{s k}^{i}$, then Proposition 3 implies that element $r(i, k)$ cannot belong to set $N_{i}$; consequently, we exclude it. After this modification, the interval thresholds can be calculated very easily:

$$
\begin{aligned}
& w_{k}^{i-}= \begin{cases}0, & k=0 \\
\bar{w}_{r(i, k-1), r(i, k)}^{i}, & k=1, \cdots,\left|N_{i}\right|-1\end{cases} \\
& w_{k}^{i+}= \begin{cases}\bar{w}_{r(i, k), r(i, k+1)}^{i} & k=0, \cdots,\left|N_{i}\right|-2 \\
\infty, & k=\left|N_{i}\right|-1\end{cases}
\end{aligned}
$$

We further define

$$
\begin{aligned}
& \varpi_{k}^{i-}=w_{k}^{i-}+\xi_{i, r(i, k)} \\
& \varpi_{k}^{i+}=w_{k}^{i+}+\xi_{i, r(i, k)}
\end{aligned}
$$

for all $i \in I, 0 \leq k \leq\left|N_{i}\right|-1$. For each $i \in I$, since $w_{k}^{i+} \leq w_{k+1}^{i-}$ and $\xi_{i, r(i, k)}<\xi_{i, r(i, k+1)}$, the intervals $\left[\varpi_{k}^{i-}, \varpi_{k}^{i+}\right]$ for $0 \leq k \leq\left|N_{i}\right|-1$ are mutually disjoint. The subproblem $\left(\mathrm{RP}_{j}\right)$ can be solved by the following algorithm:

1) For all $i \in I$, compute $N_{i}$ and intervals $\left[\varpi_{k}^{i-}, \varpi_{k}^{i+}\right], 0 \leq k \leq\left|N_{i}\right|-1$.

2) Find all possible combinations $\left\{k^{\prime}(i)\right\}_{\forall i \in I}$ for which the intersections $\bigcap_{\forall i \in I}\left[\varpi_{k^{\prime}(i)}^{i-}, \varpi_{k^{\prime}(i)}^{i+}\right]$ are nonempty. Clearly there is a polynomial number of such intersections. For each intersection, we set $z_{i r}=1$ if $r=r\left(i, k^{\prime}(i)\right)$ and 0 otherwise; further, if $w=\sum_{i \in I} \sum_{r=0}^{R} \xi_{i r} z_{i r} \in \bigcap_{\forall i \in I}\left[\varpi_{k^{\prime}(i)}^{i-}, \varpi_{k^{\prime}(i)}^{i+}\right]$, then a candidate solution is obtained.

3) From among the candidate solutions found in step 2), the one with minimum objective (28) gives the exact optimal solution.

The benefit of opening facility $j$ is given by $\phi_{j}(\boldsymbol{\lambda})=\min \left\{0, \bar{\phi}_{j}(\boldsymbol{\lambda})+f_{j}\right\}$. If $\bar{\phi}_{j}(\boldsymbol{\lambda})+f_{j}>0$, we set $y_{j}=0$ and $z_{i j r}=0, \forall i \in I, 0 \leq r \leq R$. Otherwise, we set $y_{j}=1$ and $z_{i j r}$ to the value found using the procedures above. Therefore, a lower bound for the HRLMRP is given by

$$
\phi(\lambda)=\sum_{j \in J_{e}} \phi_{j}(\boldsymbol{\lambda})+\sum_{i \in I} \sum_{r=0}^{R} \lambda_{i r} .
$$

\subsection{Upper Bound}

At each iteration of the Lagrangian relaxation procedure, we obtain an upper bound by constructing a feasible solution to the (HRLMRP). In this solution, we open the facilities that were open in the 
solution to the Lagrangian subproblem, (HRLMRP-LR'). The question then is how to assign customers to these facilities at each assignment level.

If the inventory holding cost equals 0 and if the facility disruption probabilities are identical, then the problem reduces to the reliable location models proposed by Snyder and Daskin (2005). In that problem, it is always optimal to assign a customer to open facilities level by level in increasing order of distance - that is, to assign a customer to closer facilities at lower levels $r$. However, assigning customers based solely on proximity is no longer optimal either when the disruption probabilities are heterogeneous (Cui et al. 2010) or when the inventory costs are non-zero (Chen et al. 2011). Therefore, we propose a heuristic to assign customers to open facilities.

Our heuristic begins with the solution $(\boldsymbol{y}, \boldsymbol{z})$ from $(\text { HRLMRP-LR })^{\prime}$. This solution may violate constraints (7), which require each customer to be assigned to exactly one facility at each level $r$, unless the customer is assigned to the dummy facility $e$ at a level smaller than $r$. The heuristic procedure consists of two phases: initialization and improvement. The initialization phase converts $(\boldsymbol{y}, \boldsymbol{z})$ into a solution that is feasible for $(7)$, while the improvement phase attempts to perform local updates to improve the solution. Each of these phases consists of three sub-heuristics.

Initialization Phase. In the first initialization sub-heuristic, which we call Init1, we first search for $i \in I$ and $r \in 0, \cdots, R$ such that $\sum_{j \in J} z_{i j r}+\sum_{s=0}^{r} z_{i e s}>1$ (i.e., $i$ is assigned to more than one facility $j$ at level $r$ ). For each such $i$ and $r$, we assign $i$ at level $r$ to the facility $j \in J_{e}$ that results in the smallest increase in cost. If that facility is the dummy facility $e$, then we set $z_{i j s}=0$ for all $s=r+1, \cdots, R$. Next, for each $i \in I$ and $r \in 0, \cdots, R$ such that $\sum_{j \in J} z_{i j r}+\sum_{s=0}^{r} z_{i e s}=0$ (i.e., $i$ is not assigned to any facility at level $r$ ), we assign $i$ to the $j$ that results in the smallest increase in cost. Again, if that facility is the dummy facility $e$, we set $z_{i j s}=0$ for all $s=r+1, \cdots, R$. In both cases, we loop through customers in decreasing order of their mean demands and loop through the levels in increasing order of $r$.

The second initialization sub-heuristic, Init2, simply assigns customers to facilities level by level in increasing order of distance, until it is assigned to facility $e$. The third sub-heuristic, Init3, assigns customers based purely on the disruption probabilities of the open facilities: Customers are assigned to the facility with smaller disruption probabilities at lower levels, regardless of geography.

At each iteration, we run all the three initialization sub-heuristics and choose the solution with the smallest objective value.

Improvement Phase. We propose three improvement sub-heuristics. The LevelExchange subheuristic is run each time the solution produced by the initialization phase has objective value $1.2 U B$ 
or less, where $U B$ is the objective value of the best known feasible solution. LevelExchange involves swapping the assignments at two levels, if doing so reduces the total cost. That is, if customer $i$ is assigned to facility $j_{1}$ at level $r_{1}$ and to $j_{2}$ at level $r_{2}$, then we consider swapping the assignments so that $i$ is assigned to $j_{1}$ at level $r_{2}$ and $j_{2}$ at level $r_{1}$. We search for LevelExchange moves for each customer $i$ and each level $r$, up to the level at which $i$ is assigned to facility $e$.

The LevelExchange+ sub-heuristic is an upgrade of LevelExchange. Roughly two LevelExchanges are executed in this heuristic. For a given customer $i$, we first swap the facility assignments at two levels, and then reassign $i$ at either of the two levels to another facility if doing so reduces the cost.

The third improvement sub-heuristic, DCExchange, searches for a closed facility $j_{1}$ to open and an open facility $j_{2}$ to close that will result in a reduction in cost. In particular, for each open $j_{2}$, we consider the $p$ facilities $j_{1}$ that are closest to $j_{2}$ as candidate facilities to open in place of $j_{2}$. Here, $p$ is a parameter of the algorithm; we use $p=8$. When we swap the two facilities, we reassign all customers currently assigned to $j_{2}$ to their nearest open facility (including $j_{1}$ ), and we reassign any customer to $j_{1}$ if $j_{1}$ is closer than the customer's current facility. These assignments are made level by level for each affected customer, based on distance only, not considering inventory costs. Note that $j_{1}$ is not eligible to be opened $\left[j_{2}\right.$ is not eligible to be closed] if it is fixed to 0 [1] by the variable-fixing method or the branch-and-bound process.

Because LevelExchange+ and DCExchange are more computationally intensive, we only execute them every 10 times we find a solution whose objective value is $1.2 U B$ or less.

\subsection{Initial Multipliers}

As in Snyder and Daskin (2005), to develop a strategy for computing good starting multipliers, we first examined the final multipliers for instances that have been solved to optimality. We found that the final multipliers for $r=0$ were roughly one-sixth of the demand-weighted distance from each customer to its assigned facility, and that the optimal multipliers roughly halve as $r$ increases. Therefore, in our algorithm, multipliers are initialized as

$$
\lambda_{i r}= \begin{cases}\mu_{i} \bar{c} /\left(3 \cdot 2^{r+1}\right), & r=0,1, \cdots, R-1 \\ 0, & r=R,\end{cases}
$$

where $\bar{c}=\sum_{i \in I} \sum_{j \in J} c_{i j} /|I||J|$ is the average distance between customer-DC pairs. 


\subsection{Multiplier Updating}

Each value of $\boldsymbol{\lambda}$ provides a lower bound, $\phi(\boldsymbol{\lambda})$, on the optimal objective value of (HRLMRP). To find the best possible lower bound, we need to solve

$$
\max _{\boldsymbol{\lambda}} \phi(\boldsymbol{\lambda})
$$

This problem is solved approximately using subgradient optimization. In particular, at each iteration $n$, we compute a step-size $t^{n}$ as

$$
t^{n}=\frac{\tau^{n}\left(U B-\phi\left(\lambda^{n}\right)\right)}{\sum_{i \in I} \sum_{r=0}^{R}\left(1-\sum_{j \in J} z_{i j r}-\sum_{s=0}^{r} z_{i e s}\right)^{2}},
$$

where $\tau^{n}$ is a constant at iteration $n$, initialized to 2 and halved when 20 consecutive iterations fail to improve the lower bound; $\phi\left(\boldsymbol{\lambda}^{n}\right)$ is the lower bound found at iteration $n$; and UB is the best known upper bound. The multipliers are updated by setting

$$
\lambda_{i r}^{n+1}=\lambda_{i r}^{n}+t^{n}\left(1-\sum_{j \in J} z_{i j r}-\sum_{s=0}^{r} z_{i e s}\right) .
$$

\subsection{Variable Fixing}

As suggested by Daskin et al. (2002) and Snyder and Daskin (2005), we employ the following variable fixing technique. Let $U B$ be the best known upper bound at a given iteration of the Lagrangian process. Recall that $\bar{\phi}$ is the benefit of opening facility $j\left(\$ 5.1\right.$. Suppose that $y_{j}=0$ in the solution to $\left(\mathrm{RP}_{j}^{\prime}\right)$ using $\boldsymbol{\lambda}$. If $\phi(\boldsymbol{\lambda})+f_{j}+\bar{\phi}_{j}(\boldsymbol{\lambda})>U B$, then candidate facility $j$ cannot be part of the optimal solution and we can fix $y_{j}=0$. Similarly, if $y_{j}=1$ in the solution to $\left(\mathrm{RP}_{j}^{\prime}\right)$ using $\boldsymbol{\lambda}$ and $\phi(\boldsymbol{\lambda})-\left(f_{j}+\bar{\phi}_{j}(\boldsymbol{\lambda})\right)>U B$, then candidate facility $j$ must be part of the optimal solution and we can fix $y_{j}=1$. The time required to perform these checks is negligible. We refer the reader to Daskin et al. (2002), Snyder and Daskin (2005), or Snyder and Shen (2011, §7.2.4.6) for justification of these rules.

Rather than performing these checks only at the end of the Lagrangian procedure at the root node, as Daskin et al. (2002) and Snyder and Daskin (2005) do, we perform the variable-fixing checks at each iteration of the Lagrangian procedure, and at each node of the branch and bound tree. In our problem, the slight increase in computational effort required to perform the check at every iteration is warranted since $\boldsymbol{Q}$ is a more accurate approximation for $\boldsymbol{p}$ when more variables are forced in or out of the solution. 


\subsection{Branch and Bound}

If the Lagrangian process terminates with the lower bound and best known upper bound equal (to within some pre-specified tolerance), we have found the optimal solution to (HRLMRP). If, however, the lower bound is less than the best known upper bound and some candidate facility locations are not forced into or out of the solution, which is often the case in our problem because of our approximation for $\boldsymbol{p}$, one can employ a branch and bound procedure to close the gap, with branching performed on the $\boldsymbol{y}$ (location) variables.

At each node of the branch and bound tree, we first try to branch on a location variable corresponding to a facility that is in the best known feasible solution. From among these locations, we select the unfixed facility location with the largest assigned demand. If all facilities in the solution have already been forced into the solution, then we branch on the first unforced facility. The selected variable is first fixed to 0 and then to 1 . Branching is done in a depth-first manner. The tree is fathomed at a given node if the lower bound at that node is greater than or equal to the objective value of the best feasible solution found anywhere in the tree to date, or if all facilities have been forced open or closed. The final Lagrangian multipliers at a given node are passed to its child nodes and are used as initial multipliers at those nodes.

Unfortunately, we have found the branch-and-bound procedure to be relatively ineffective, as discussed in 6.4 . Therefore, in our computational experiments, we report root-node results only.

\section{Numerical Experiments}

We tested the SOS2 and LR methods on benchmark instances based on U.S. census data: a 49-node data set consisting of Washington D.C. and the 48 continental state capitals, an 88-node set consisting of the union of the 49-node set and the 50 largest cities in the United States, and a 150-node set consisting of the 150 largest cities in the United States. These data sets are based on those by Daskin (2013) and modified for the RFLP by Snyder and Daskin (2005) 2 We implemented our proposed $\mathrm{LR}$ algorithm in the $\mathrm{C} \#$ programming language and ran it on a $\mathrm{PC}$ with a $2.3 \mathrm{GHz} \mathrm{AMD}$ Sempron Processor and 2 GB memory. We solved the SOS2 model using Gurobi 5.6. In the LR algorithm, we set the optimality tolerance $\varepsilon=0.1 \%$; the algorithm also terminates if $\tau^{n}<10^{-5}$, or if the number of iterations exceeds 2000. For SOS2, we used $|V|=100$ breakpoints and set a time limit for the solver of $t_{m}=3600$ s for all instances.

\footnotetext{
${ }^{2}$ The Snyder and Daskin $(2005)$ data sets are available at http://coral.ie.lehigh.edu/ larry/research/publications/.
} 
In this section, we first demonstrate the accuracy of the approximation of the inventory cost ( 3.2 .1 . Then we conduct three sets of numerical experiments to test the proposed model and solution approaches. Since there are no reported algorithms against which to compare our methods for the HRLMRP, we first compare our methods to published algorithms for two special cases - the RFLP with heterogeneous $\boldsymbol{q}$ and the RLMRP with identical $\boldsymbol{q}$. Then, our third experiment evaluates the general model proposed in this paper, the HRLMRP with heterogeneous $\boldsymbol{q}$.

In all the tables that follow, columns labeled "gap" are in \%, those labeled "time" are in seconds, and those labeled "DC" indicate the number of opened facilities in the corresponding solution. In a given row, the best known solution(s), from either our proposed approaches or published results, are marked in boldface. In addition, the weight assigned to the inventory cost, $\theta$, is set to 1 unless otherwise specified.

\subsection{Accuracy of Inventory Cost Approximation}

Equation (4) gives an approximation of the exact inventory cost function (3). To confirm the accuracy of this approximation, we conduct a simulation experiment, as follows:

1. Randomly generate a problem instance with 10 potential DC locations and 30 customers. Customer demand means $\mu_{i}$ are drawn uniformly from $[50,80]$ and parameters $K_{j}$ are drawn from [10,30]. Facility disruption probabilities $q_{j}$ are drawn from $[0.005,0.035]$.

2. Randomly generate a feasible solution $(\boldsymbol{y}, \boldsymbol{z})$.

3. Use (3) to calculate the exact expected inventory cost $C$. This requires enumerating all $2^{|J|}$ possible subsets $J^{\prime}$ of $J$.

4. Use (4) to calculate the approximate expected inventory $\operatorname{cost} C^{\prime}$ and calculate the approximation error.

5. Repeat steps 2-4 100 times.

For the 100 randomly generated feasible solutions, Figure 1(a) plots the approximate cost $C^{\prime}$ vs. the exact cost $C$. The mean error across the 100 solutions is only $0.776 \%$. We also repeated steps $1-$ 5100 times to generate 100 instances, each with 100 solutions. The resulting 10,000 solutions are plotted in Figure 1(b), with a mean error of $0.852 \%$. The maximum errors of the 100- and 10,000solution experiments are $1.108 \%$ and $1.223 \%$, respectively. This confirms that our approximation is quite accurate and justifies its use in our models. 


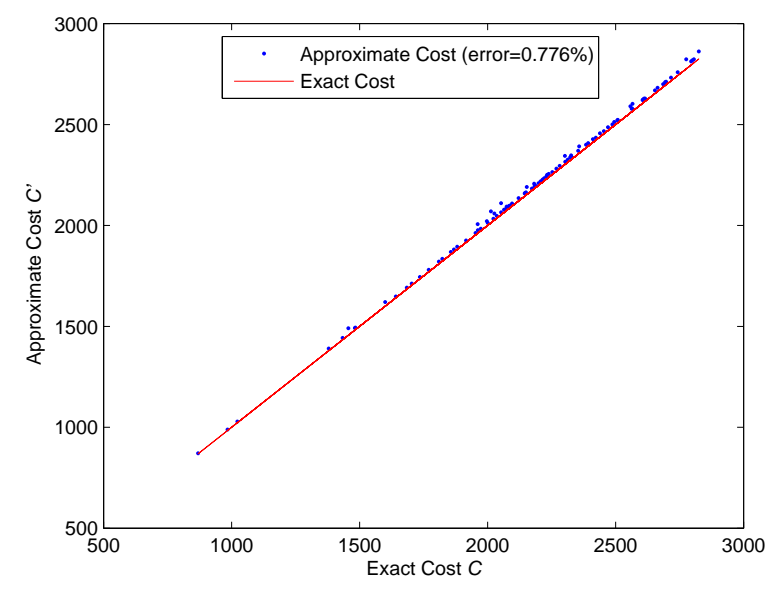

(a) One instance, 100 solutions

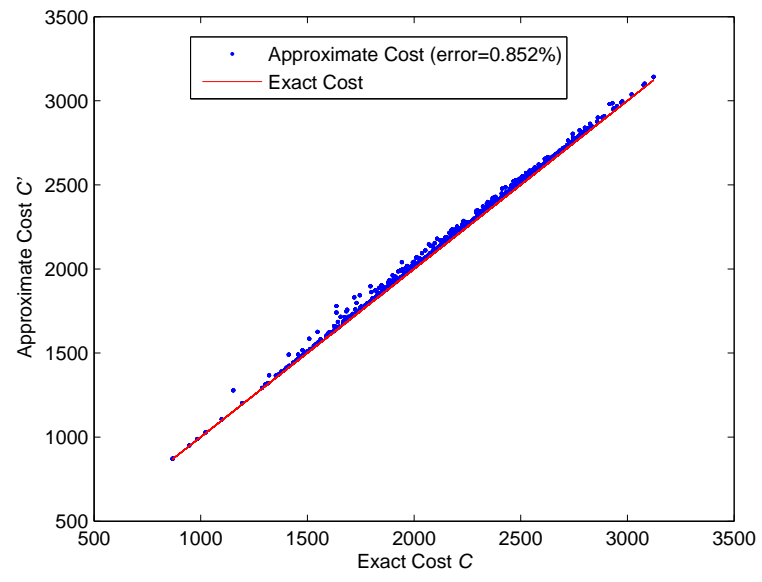

(b) 100 instances, with 100 solutions for each

Figure 1: Approximate vs. exact expected inventory cost

\subsection{RFLP with Heterogenous $q$}

This section evaluates the performance of our proposed solution methods by implementing them for a special case, the RFLP with heterogeneous $\boldsymbol{q}$, and comparing them to the results reported by Cui et al. (2010) for their LR algorithm. Our goal is not to argue that our method should be used instead of theirs for this special case (since our algorithm is more general and theirs is tailored for the specific problem), but rather to show that ours is competitive with theirs and to justify its performance when compared to published results.

Cui et al. (2010) report results for the 49- and 88-node data sets, but not for the 150-node set. They also report results for the 50- and 100-node Euclidean instances by Snyder and Daskin (2005), for which they generate random $q_{j}$ values; however, we did not have access to their randomly generated $q_{j}$ values and so we cannot provide a comparison on those data sets.

As in Cui et al. (2010), we set the shipment cost $c_{i j}$ to the great circle distance (in miles) between locations $i$ and $j$. The failure probabilities $q_{j}$ are calculated as $q_{j}=0.1 e^{-l_{j} / 400}$, where $l_{j}$ is the great circle distance (in miles) between location $j$ and New Orleans, LA. Also, we ignore the inventory cost and the orders placed by facilities to the supplier, so we set $g_{j}=a_{j}=F_{j}=h_{j}=L=0, \beta=1$, and $\theta=0$ for all $j \in J$.

For each data set, the results of LR and SOS2 for $R=2,3,4$ are shown in Table 1. LR solved all instances in less than 1 minutes (roughly), much faster than the LR algorithm by Cui et al. (2010). In addition, the proposed LR heuristic found better solutions than Cui et al. (2010) for three instances, 
with less computation time. (Note that the "gap" columns refer to the gap between the upper and lower bounds found by the individual algorithms, not between the upper bound and the optimal objective value.) The larger gaps for our LR heuristic result from our use of the lower bound $\boldsymbol{Q}$ in place of $\boldsymbol{p}$. (How to close this gap is an interesting issue for future research.) The last three columns indicate that SOS2 can find the optimal solution for small-scale instances, but due to the time limit and insufficient memory, SOS2 terminates with a gap for larger instances.

Table 1: Numerical results for RFLP with heterogenous $\boldsymbol{q}$

\begin{tabular}{|c|c|c|c|c|c|c|c|c|c|c|c|}
\hline \multirow[b]{2}{*}{ Nodes } & \multirow[b]{2}{*}{$R$} & \multicolumn{3}{|c|}{ Cui et al. (Overall) } & \multicolumn{4}{|c|}{ LR (Root) } & \multicolumn{3}{|c|}{ SOS2 } \\
\hline & & UB & gap & time & $\mathrm{DC}$ & UB & gap & time & UB & gap & time \\
\hline 49 & 2 & 880098 & 0.141 & 9 & 6 & 881575.57 & 3.697 & 1 & 879330.63 & 0.00 & 229 \\
\hline 49 & 3 & 874423 & 0.482 & 19 & 6 & 874212.09 & 2.801 & 2 & 4181.10 & 0.08 & 3600 \\
\hline 49 & 4 & 874323 & 0.481 & 50 & 6 & 874086.34 & 2.861 & 3 & 874084.79 & 0.04 & 3600 \\
\hline 88 & 2 & 1234860 & 0.385 & 66 & 9 & 1233954.70 & 3.414 & 17 & 1233885.65 & 0.22 & 3600 \\
\hline 88 & 3 & 1223610 & 0.489 & 458 & 9 & 1224190.58 & 2.788 & 15 & 1224102.92 & 0.10 & 3600 \\
\hline 88 & 4 & 1223290 & 0.494 & 883 & 9 & 1223919.63 & 2.633 & 22 & 1224112.12 & 0.76 & 3600 \\
\hline 150 & 2 & - & - & - & 7 & 1718330.05 & 3.728 & 41 & 1720722.31 & 3.43 & 3600 \\
\hline 150 & 3 & - & - & - & 7 & 1703408.06 & 3.033 & 38 & 1714935.59 & 3.01 & 3600 \\
\hline 150 & 4 & - & - & - & 7 & 1703177.10 & 2.933 & 61 & 1723731.56 & 3.85 & 3600 \\
\hline
\end{tabular}

The maximum assignment level $R$ does not affect the optimal facility locations in any of our test instances, although a larger $R$ in general leads to a smaller optimal cost, which is consistent with the conclusion in Cui et al. (2010).

\subsection{RLMRP with Identical $q$}

In Chen et al. (2011), all facilities are assumed to fail independently with an identical probability $q$. In this section, we test our algorithms for this special case, and compare to them. Chen et al. (2011) report results for the 49-, 88- and 150-node data sets. The shipment cost $c_{i j}$ between any two locations is proportional to the great circle distance (in $\mathrm{km}$ ) by a factor $s$. The holding $\operatorname{cost} h_{j}$ is equal to $10^{-3} f_{j}$ for each facility. Customers face a constant demand. Other parameter settings are $F_{j}=1000, a_{j}=5, \pi_{i}=100$.

We test both LR and SOS2 for the 49-node and 88-node dataset:3 and compare with Chen et al. (2011). LR can be modified to solve this problem by simply setting all $\hat{c}_{i j r}=\infty$ if $j=|J|$ and $r<R$.

\footnotetext{
${ }^{3}$ The results reported in Table 4 of Chen et al. (2011) appear to be incorrect for the 150-node data set. In this data set, each facility has a fixed cost of 10000 . However, for test 1 , for example, the fixed cost is $198511 \times 20.654 \%=41000$; however, 5 facilities are open, so the fixed set-up cost should be 50000. Therefore, we do not compare our results to theirs for the 150-node data set.
} 
The results are shown in Table 2 and Table 3, respectively. As in Table 1, the "gap" columns refer to the gap between the upper and lower bounds found by the individual algorithms. We see that LR can solve all these instances to very tight optimality gaps (less than $1 \%$ for all instances) in a short time (in 20 seconds for the 49-node dataset, and in 150 seconds for the 88-node dataset). Compared with Chen et al. (2011), among all 56 instances, LR found 36 better solutions and obtained the same solutions in the other 20 instances. Moreover, LR needs much less computational time.

SOS2 can find the optimal solutions for 15 instances of the 49-node dataset, while the optimality gaps are less than $0.04 \%$ for the other 13 instances of the 49 -node dataset, and less than $0.05 \%$ for all the instances in the 88-node dataset. More promisingly, SOS2 can find 51 better solutions compared with Chen et al. (2011). These results lead us to believe that, if enough time is allowed, SOS2 would be able to find the optimal solutions for all instances.

Although our method to solve the subproblems is based on Chen et al. (2011), our improvement in calculating the interval thresholds, as well as the heuristic to get the upper bound, makes LR more competitive for the RLMRP with identical $\boldsymbol{q}$, which motivates us next to test the performance in the heterogeneous case.

\subsection{HRLMRP with Heterogenous $q$}

In this section, we test a series of instances in which the failure probabilities may be different among the facilities. We test our algorithms using the U.S. census data sets described above. For each facility $j \in J$, the unit holding cost is $h_{j}=10$, the fixed cost when placing an order to the supplier is $F_{j}=1000$, and the fixed cost and volume-dependent cost of a shipment are $g_{j}=10$ and $a_{j}=5$, respectively. Customers face normally distributed demands, with $\gamma=1$ and $z_{\alpha}=1.96$ to ensure the service level. For each data set, the parameters may take the following values: $R \in\{1,2,3,4,5\}$, $L \in\{1,3,5,7,9\}$, and $\beta \in\{0.2,0.6,1.0\}$. The results for the 49-node data set are shown in Table 4 . In total, we generated 42 instances. The third column is labeled as " $E(q)-n$ "; this indicates that the failure probability is drawn uniformly from the interval $[(1-0.1 n) E(q),(1+0.1 n) E(q)]$ (so the mean value is $E(q))$.

In Table 4, the last column "DIFF" gives the relative differences between the solution found by LR and the solution obtained by SOS2. "In" and "Out" mean the number of DCs that are forced in or out at the final solutions; the four columns under "Cost component (\%)" represent the cost percentage of the fixed setup cost, inventory cost, shipment cost and penalty cost, respectively. The following conclusions can be drawn from Table 4 
Table 2: Numerical results for the 49-node dataset (RLMRP, identical $\boldsymbol{q}$ )

\begin{tabular}{|c|c|c|c|c|c|c|c|c|c|c|c|c|c|}
\hline \multirow[b]{2}{*}{ No. } & \multirow[b]{2}{*}{ Node } & \multirow[b]{2}{*}{$R$} & \multirow[b]{2}{*}{$s$} & \multirow[b]{2}{*}{$q$} & \multicolumn{3}{|c|}{ Chen et al. } & \multicolumn{3}{|c|}{$\mathrm{LR}$} & \multicolumn{3}{|c|}{ SOS2 } \\
\hline & & & & & UB & gap & time & UB & gap & time & UB & gap & time \\
\hline 1 & 49 & 3 & 0.05 & 0.1 & 111153 & 0.190 & 17.9 & 111152 & 0.010 & 7.5 & 111142 & 0.000 & 314.1 \\
\hline 2 & 49 & 3 & 0.05 & 0.3 & 133381 & 0.990 & 53.9 & 132327 & 0.184 & 17.4 & 132289 & 0.000 & 3469.4 \\
\hline 3 & 49 & 3 & 0.05 & 0.5 & 159592 & 0.921 & 23.6 & 159202 & 0.010 & 9.3 & 159150 & 0.000 & 3529.9 \\
\hline 4 & 49 & 3 & 0.10 & 0.1 & 154537 & 0.903 & 23.1 & 154537 & 0.010 & 11.5 & 154500 & 0.006 & 3600.0 \\
\hline 5 & 49 & 3 & 0.10 & 0.3 & 183921 & 0.770 & 14.6 & 183620 & 0.167 & 13.4 & 183661 & 0.041 & 3600.0 \\
\hline 6 & 49 & 3 & 0.10 & 0.5 & 213984 & 0.501 & 17.8 & 213984 & 0.425 & 18.0 & 213902 & 0.035 & 3600.0 \\
\hline 7 & 49 & 5 & 0.05 & 0.1 & 111179 & 0.380 & 37.3 & 111178 & 0.009 & 11.9 & 111168 & 0.000 & 836.0 \\
\hline 8 & 49 & 5 & 0.05 & 0.3 & 132927 & 0.935 & 37.6 & 131844 & 0.000 & 15.7 & 131798 & 0.008 & 3600.0 \\
\hline 9 & 49 & 5 & 0.05 & 0.5 & 158375 & 0.988 & 50.7 & 157351 & 0.010 & 9.9 & 157817 & 0.026 & 3600.0 \\
\hline 10 & 49 & 5 & 0.10 & 0.1 & 154674 & 0.913 & 32.2 & 154674 & 0.011 & 15.7 & 154798 & 0.013 & 3600.0 \\
\hline 11 & 49 & 5 & 0.10 & 0.3 & 186758 & 0.777 & 26.3 & 186413 & 0.590 & 19.3 & 186477 & 0.039 & 3600.0 \\
\hline 12 & 49 & 5 & 0.10 & 0.5 & 223796 & 0.986 & 18.0 & 222385 & 0.578 & 18.8 & 222240 & 0.031 & 3600.0 \\
\hline 13 & 49 & 2 & 0.05 & 0.1 & 111517 & 0.239 & 21.4 & 111512 & 0.005 & 8.4 & 111498 & 0.000 & 279.2 \\
\hline 14 & 49 & 4 & 0.05 & 0.1 & 111164 & 0.963 & 26.8 & 111163 & 0.007 & 9.9 & 111153 & 0.000 & 743.7 \\
\hline 15 & 49 & 6 & 0.05 & 0.1 & 112127 & 0.422 & 64.1 & 111666 & 0.010 & 15.9 & 112097 & 0.000 & 2531.2 \\
\hline 16 & 49 & 3 & 0.15 & 0.1 & 186683 & 0.700 & 11.0 & 186682 & 0.138 & 8.0 & 186896 & 0.020 & 3600.0 \\
\hline 17 & 49 & 3 & 0.20 & 0.1 & 212366 & 0.868 & 8.2 & 212366 & 0.199 & 14.4 & 211965 & 0.018 & 3600.0 \\
\hline 18 & 49 & 1 & 0.05 & 0.1 & 118626 & 0.061 & 10.5 & 118625 & 0.002 & 5.0 & 118615 & 0.000 & 63.1 \\
\hline 19 & 49 & 1 & 0.05 & 0.3 & 153816 & 0.152 & 8.7 & 153816 & 0.010 & 3.1 & 153802 & 0.000 & 23.7 \\
\hline 20 & 49 & 1 & 0.05 & 0.5 & 188552 & 0.898 & 4.9 & 188150 & 0.119 & 2.3 & 188117 & 0.000 & 125.5 \\
\hline 21 & 49 & 1 & 0.10 & 0.1 & 155193 & 0.395 & 10.1 & 155173 & 0.242 & 2.5 & 155141 & 0.000 & 79.6 \\
\hline 22 & 49 & 1 & 0.10 & 0.3 & 186447 & 0.789 & 6.1 & 185345 & 0.066 & 4.9 & 185316 & 0.000 & 169.6 \\
\hline 23 & 49 & 1 & 0.10 & 0.5 & 214250 & 0.063 & 21.4 & 214250 & 0.010 & 3.0 & 214195 & 0.000 & 389.3 \\
\hline 24 & 49 & 2 & 0.05 & 0.3 & 135984 & 0.696 & 14.9 & 135605 & 0.288 & 7.3 & 135565 & 0.000 & 1608.3 \\
\hline 25 & 49 & 2 & 0.05 & 0.5 & 167756 & 0.956 & 15.7 & 166705 & 0.031 & 9.5 & 166668 & 0.000 & 2189.8 \\
\hline 26 & 49 & 2 & 0.10 & 0.1 & 153745 & 0.217 & 17.7 & 153745 & 0.016 & 14.3 & 153713 & 0.007 & 3600.0 \\
\hline 27 & 49 & 2 & 0.10 & 0.3 & 180496 & 0.683 & 14.5 & 179786 & 0.036 & 15.6 & 179740 & 0.030 & 3600.0 \\
\hline 28 & 49 & 2 & 0.10 & 0.5 & 209709 & 0.067 & 17.5 & 209709 & 0.140 & 9.5 & 209627 & 0.026 & 3600.0 \\
\hline Avg. & - & - & - & - & 161525 & 0.622 & 22.4 & 161177 & 0.119 & 10.8 & 161180 & 0.011 & 2255.4 \\
\hline
\end{tabular}

1. From \# 1-5 and 6-10, it is clear that the total cost increases dramatically with $\boldsymbol{q}$, due to the enormous additional cost incurred by customer reassignments.

2. In \# $1-5, R=1$, which is very small. In these instances, $\boldsymbol{q}$ has an insignificant influence on the optimal number of facilities; however, in instances $6-10$, in which $R=5$, the optimal number of facilities increases with $\boldsymbol{q}$. This is because if the failure probability is large, additional facilities can provide better redundancy for reliable service quality against facility failures. If customers can be assigned to more backup facilities, there is more benefit and therefore more potential to offset the fixed cost of locating additional facilities.

3. \# 11-15 show that the total cost decreases as we allow more backup assignments.

4. \# 16-18 and 19-21 illustrate that the number of facilities in the optimal solution increases with $\beta$, i.e., as the weight on the transportation cost increases compared to that on the inventory cost, in order to reduce the distance travelled. This means that the transportation cost gets 
Table 3: Numerical results for the 88-node dataset (RLMRP, identical $\boldsymbol{q}$ )

\begin{tabular}{|c|c|c|c|c|c|c|c|c|c|c|c|c|c|}
\hline \multirow[b]{2}{*}{ No. } & \multirow[b]{2}{*}{ Node } & \multirow[b]{2}{*}{$R$} & \multirow[b]{2}{*}{$s$} & \multirow[b]{2}{*}{$q$} & \multicolumn{3}{|c|}{ Chen et al. } & \multicolumn{3}{|c|}{ LR } & \multicolumn{3}{|c|}{ SOS2 } \\
\hline & & & & & UB & gap & time & UB & gap & time & UB & gap & time \\
\hline 1 & 88 & 3 & 0.05 & 0.1 & 149300 & 0.790 & 84.5 & 149300 & 0.067 & 61.6 & 149287 & 0.022 & 3600.0 \\
\hline 2 & 88 & 3 & 0.05 & 0.3 & 176204 & 0.916 & 81.3 & 176022 & 0.170 & 50.2 & 175995 & 0.027 & 3600.0 \\
\hline 3 & 88 & 3 & 0.05 & 0.5 & 222010 & 0.526 & 108.6 & 222010 & 0.755 & 73.1 & 221978 & 0.030 & 3600.0 \\
\hline 4 & 88 & 3 & 0.10 & 0.1 & 209612 & 0.802 & 61.1 & 209610 & 0.381 & 36.4 & 209547 & 0.031 & 3600.0 \\
\hline 5 & 88 & 3 & 0.10 & 0.3 & 245679 & 0.975 & 65.6 & 245395 & 0.522 & 78.6 & 245329 & 0.027 & 3600.0 \\
\hline 6 & 88 & 3 & 0.10 & 0.5 & 293315 & 0.843 & 49.2 & 293190 & 0.925 & 84.7 & 292973 & 0.037 & 3600.0 \\
\hline 7 & 88 & 5 & 0.05 & 0.1 & 149248 & 0.984 & 183.9 & 149248 & 0.026 & 125.3 & 149235 & 0.030 & 3600.0 \\
\hline 8 & 88 & 5 & 0.05 & 0.3 & 173345 & 0.948 & 174.1 & 172793 & 0.215 & 131.8 & 172747 & 0.037 & 3600.0 \\
\hline 9 & 88 & 5 & 0.05 & 0.5 & 207233 & 0.878 & 188.3 & 207227 & 0.231 & 119.2 & 207336 & 0.041 & 3600.0 \\
\hline 10 & 88 & 5 & 0.10 & 0.1 & 209696 & 0.953 & 96.4 & 209694 & 0.986 & 103.1 & 209631 & 0.041 & 3600.0 \\
\hline 11 & 88 & 5 & 0.10 & 0.3 & 246432 & 0.888 & 90.0 & 246431 & 0.416 & 154.9 & 246381 & 0.033 & 3600.0 \\
\hline 12 & 88 & 5 & 0.10 & 0.5 & 291527 & 0.861 & 73.4 & 291526 & 0.931 & 51.7 & 291398 & 0.041 & 3600.0 \\
\hline 13 & 88 & 2 & 0.05 & 0.1 & 150457 & 0.687 & 39.5 & 150457 & 0.010 & 29.8 & 150446 & 0.012 & 3600.0 \\
\hline 14 & 88 & 4 & 0.05 & 0.1 & 149242 & 0.987 & 121.9 & 149242 & 0.010 & 36.6 & 149502 & 0.027 & 3600.0 \\
\hline 15 & 88 & 6 & 0.05 & 0.1 & 149250 & 0.898 & 278.5 & 149250 & 0.115 & 60.4 & 150658 & 0.051 & 3600.0 \\
\hline 16 & 88 & 3 & 0.15 & 0.1 & 258271 & 0.664 & 80.1 & 258270 & 0.871 & 83.8 & 258193 & 0.024 & 3600.0 \\
\hline 17 & 88 & 3 & 0.20 & 0.1 & 301808 & 0.950 & 75.1 & 301759 & 0.947 & 46.1 & 1637 & 0.031 & 3600.0 \\
\hline 18 & 88 & 1 & 0.05 & 0.1 & 170312 & 0.284 & 28.6 & 170312 & 0.010 & 29.3 & 170301 & 0.000 & 140.8 \\
\hline 19 & 88 & 1 & 0.05 & 0.3 & 240377 & 0.861 & 38.7 & 239422 & 0.053 & 30.9 & 239410 & 0.000 & 58.2 \\
\hline 20 & 88 & 1 & 0.05 & 0.5 & 307865 & 0.704 & 16.4 & 307343 & 0.037 & 14.7 & 307331 & 0.000 & 21.2 \\
\hline 21 & 88 & 1 & 0.10 & 0.1 & 223575 & 0.105 & 37.3 & 223575 & 0.046 & 38.9 & 223535 & 0.011 & 3600.0 \\
\hline 22 & 88 & 1 & 0.10 & 0.3 & 287999 & 0.920 & 42.0 & 286731 & 0.445 & 12.8 & 286544 & 0.002 & 3600.0 \\
\hline 23 & 88 & 1 & 0.10 & 0.5 & 344269 & 0.069 & 33.7 & 344269 & 0.010 & 12.2 & 344234 & 0.000 & 199.1 \\
\hline 24 & 88 & 2 & 0.05 & 0.3 & 189960 & 0.998 & 83.5 & 188678 & 0.419 & 25.4 & 188655 & 0.021 & 3600.0 \\
\hline 25 & 88 & 2 & 0.05 & 0.5 & 247971 & 0.952 & 41.4 & 247322 & 0.910 & 67.9 & 247377 & 0.025 & 3600.0 \\
\hline 26 & 88 & 2 & 0.10 & 0.1 & 209968 & 0.483 & 60.2 & 209880 & 0.844 & 56.9 & 209822 & 0.029 & 3600.0 \\
\hline 27 & 88 & 2 & 0.10 & 0.3 & 248462 & 0.857 & 41.8 & 248462 & 0.175 & 28.9 & 248309 & 0.015 & 3600.0 \\
\hline 28 & 88 & 2 & 0.10 & 0.5 & 303068 & 0.914 & 64.4 & 303068 & 0.117 & 30.8 & 302798 & 0.018 & 3600.0 \\
\hline vg. & - & - & - & - & 227016 & 0.775 & 83.6 & 226803 & 0.380 & 59.9 & 226807 & 0.024 & 3100.7 \\
\hline
\end{tabular}

more weight compared to the inventory cost, for example, see the numbers in the "Inventory" and "Shipment" columns of \# 16-18, $\frac{54.43}{3.73}=14.61<\frac{61.72}{2.28}=27.10<\frac{58.71}{2.04}=28.82$. When the shipment cost dominates, more facilities are deployed to reduce the travel distance. In contrast, when the inventory cost dominates, the optimal number of facilities decreases because inventory becomes more expensive and pooling become more attractive.

5. The lead time also affects the system cost components: The inventory cost comprises a larger proportion of the total cost when the lead time $L$ increases, as demonstrated in \# 22-26.

6. The penalty cost increases significantly with $q$ and decreases dramatically with $R$.

These rules are consistent with the identical probability case concluded in Chen et al. (2011). When the LR procedure proposed in $\$ 5$ terminates with a gap that is larger than desired, it is natural to use branch-and-bound to close this gap, as discussed in $\$ 5.7$. However, branch-and-bound is not as effective as desired, because of our approximation of $\boldsymbol{p}$ by $\boldsymbol{Q}$. Although the lower bounds from 
(HRLMRP-LR') are not much weaker than those from (HRLMRP-LR), the gap often cannot be closed without branching on the assignment variables $\boldsymbol{z}$ in addition to the location variables $\boldsymbol{y}$. Even when the solution to (HRLMRP-LR') is feasible (and therefore optimal) for the primal problem, the LB-UB gap may be non-zero. Branching on the assignment variables is impractical since there are many more assignment variables. (In contrast, for most facility location problems it is sufficient to branch only on the location variables.)

However, in our preliminary experiments using branch-and-bound we found that the best feasible solutions were often found at the root node, and that branching yields little or no improvement in solution quality, with a high computational expense. Therefore, we have not used the branch-andbound procedure here. Instead, we leave the development and improvement of such an algorithm as a topic for future research.

We also tested our proposed approaches on the 88- and 150-node datasets, resulting in mean root gaps of 12.692 and 8.974, respectively, with mean CPU times of 31.24 and 102.55 seconds, respectively. More detailed results are given in Tables A1 and A2 in the Appendix. These tables also indicate (in the "Non-closest" column) the number of customers that are not assigned to their closest DC at the primary level, i.e., at $r=0$. Because SOS2 could not solve most of the 49-node instances within the time and memory limits, we did not use SOS2 to solve the 88- and 150-node instances. The results for LR on those instances remain consistent with those from the 49-node dataset. Despite the increased problem size, the proposed Lagrangian relaxation heuristic can still solve them efficiently.

The results in Tables 4 A1 and A2 suggest that, if many DCs are forced out of the solution (e.g., \# 6, 7, 16, 35, 42 in Table 4, \# 6 in Table A1 and \# 6 in Table A2), the optimality gaps will be relatively small, which demonstrates the effectiveness of our method to update $\boldsymbol{Q}$ in $\$ 5.2 .1$.

Finally, we briefly report on two other experiments that we conducted. First, we re-solved the SOS2 model, specifying that the solver should terminate when the optimality gap reached the same gap that the LR approach obtained for each instance. The results show that the SOS2 approach takes considerably longer to reach the same gaps - thousands of seconds for SOS2 compared to less than 10 seconds for LR, on average - further justifying the use of the LR approach, especially for larger instances.

Second, recall that our heuristic in $\$ 5.3$ uses three initialization sub-heuristics and selects the best one in each iteration. We conducted an experiment to determine how often each sub-heuristic is the best. We repeated the tests above, using each sub-heuristic individually. The results show that Init3 performs well in the early iterations, while Init1 and Init2 are more promising when the multipliers 
have already improved in later iterations. This suggests that, if a modeler only wished to code one of the three sub-heuristics, she or he should choose Init3 if a small iteration limit is to be used and Init2 otherwise (since Init1 is more difficult to code than Init2). However, the best performance results from using all three sub-heuristics, as proposed in $\$ 5.3$. 


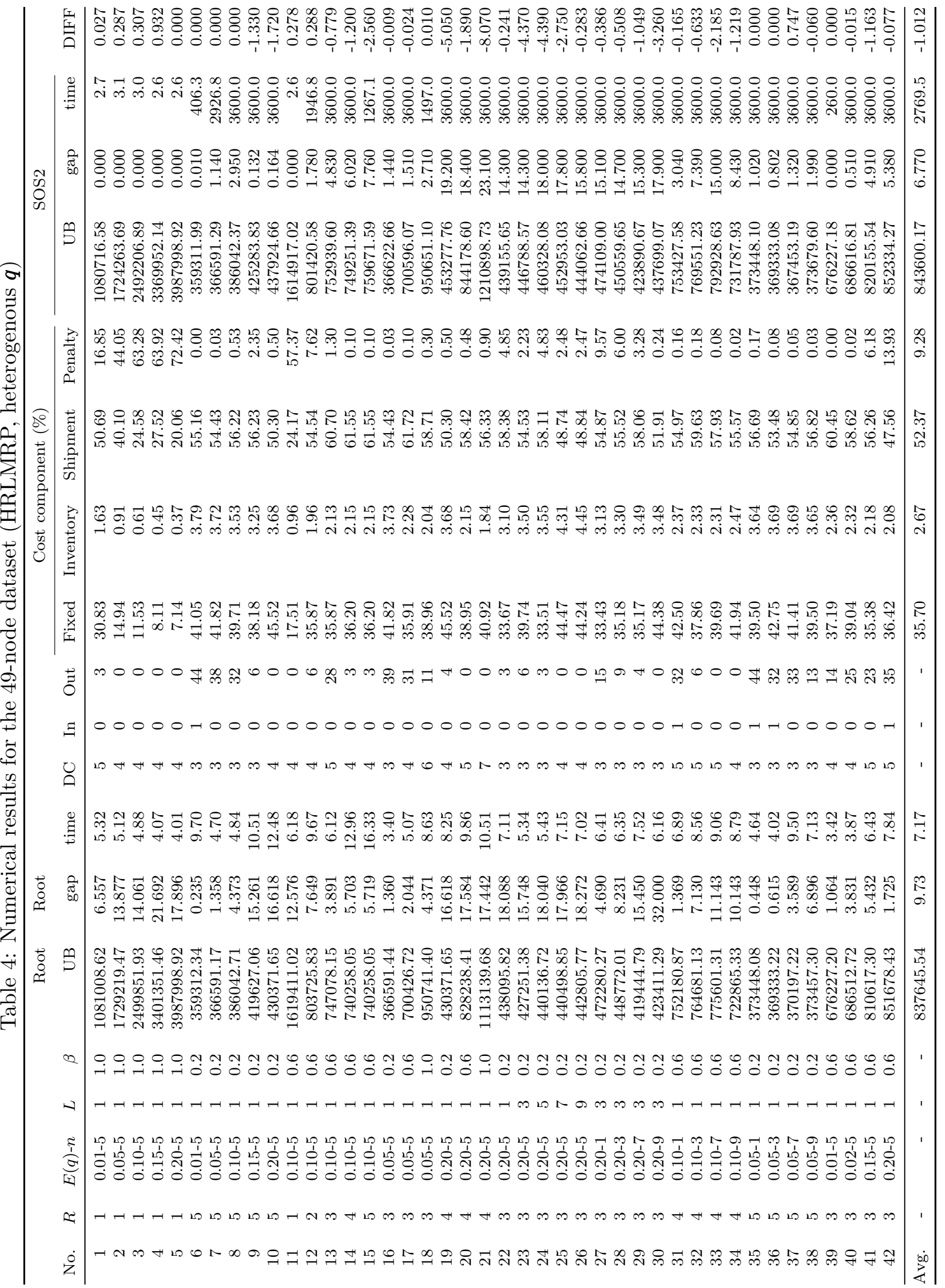




\subsection{Comparison of Heterogeneous and Identical $q$ in RLMRP}

We are also interested in comparing the solutions that result from heterogeneous and identical disruption probabilities, and the error that results if one were to use identical $q$ as a heuristic to solve a problem in which the $q_{j}$ were actually heterogeneous. To this end, we solve four groups of instances; the failure probabilities $(q)$ of each of the instances in the first three groups have the same mean value $(E(q))$, while those in the last group have the same variability $(n)$. (Recall that the notation $E(q)-n$ indicates that $q$ is drawn from the range $[(1-0.1 n) E(q),(1+0.1 n) E(q)]$.) Moreover, the first instance in each of the first three groups has homogeneous failure probabilities $(n=0)$, while in the remaining instances they are heterogeneous $(n>0)$. Table 5 reports results for instances $\# 7,13,14,17,23$, and 27-42 from Table 4, In all instances we use $\theta=1$.

For each instance we solved the HRLMRP using LR; the cost of the resulting solution is reported in the "HRLMRP Cost" column. For instances with $n>0$, we also solved the RLMRP with identical $q$, setting $q$ equal to $E(q)$, again using LR; we then used the exact (heterogeneous $q$ ) objective function to evaluate the cost of the resulting solution, to measure its quality as a heuristic. This cost is listed in the "Identical $q$ Cost" column, and the percentage difference is listed in the "DIFF" column. Clearly, solving the identical- $q$ model when the $q_{j}$ are actually heterogeneous can lead to large errors.

The last two columns indicate the number of open DCs in the HRLMRP solution and their indices. Clearly, the optimal DCs change as the variability in $q_{j}$ changes. As expected, the identical- $q$ solution results in 0 error only when the HRLMRP solution happens to have the same open facilities as the solution for $n=0$. This suggests that solving the RLMRP with $q$ set to the average of the $q_{j}$ results in a loss of accuracy, although the run time is shorter.

Figure 2 shows the optimal facility deployments and level-0 assignments under $E(q)=0.05, R=$ $5, L=1, \beta=0.2$, and $\theta=1$. The solution is the same for $n=0,3$ and 7 ; this solution is shown in Figure 2(a) also the solution is the same for $n=1$ and 9 , and is shown in Figure 2(b), The solution for $n=5$ is shown in Figure 2(c). The solutions are relatively similar across different $n$ values and do not display an obvious pattern as $n$ changes. Note, however, that the solutions differ more between different $E(q)$ values, as shown in the last 6 tests. This suggests that the optimal facility locations depend more on $E(q)$ than on $n$. 
Table 5: Solution Comparison: Heterogeneous vs. Identical $q(\theta=1)$

\begin{tabular}{|c|c|c|c|c|c|c|c|c|c|}
\hline No. & $R$ & $L$ & $\beta$ & $E(q)-n$ & HRLMRP Cost & Identical $q$ Cost & DIFF & $\mathrm{DC}$ & Open DCs \\
\hline 1 & & & & $0.20-0$ & 469155.87 & - & - & 3 & 5629 \\
\hline 2 & & & & $0.20-1$ & 472280.27 & 472280.27 & 0.000 & 3 & 5629 \\
\hline 3 & 3 & 3 & ? & $0.20-3$ & 448772.01 & 448772.00 & 0.000 & 3 & 5629 \\
\hline 4 & 3 & 3 & 0.2 & $0.20-5$ & 427251.38 & 497236.26 & 16.380 & 3 & 52932 \\
\hline 5 & & & & $0.20-7$ & 419444.79 & 450521.84 & 7.409 & 3 & 52932 \\
\hline 6 & & & & $0.20-9$ & 423411.29 & 480144.66 & 13.399 & 3 & 152934 \\
\hline 7 & & & & $0.10-0$ & 753116.02 & . & - & 5 & 58222839 \\
\hline 8 & & & & $0.10-1$ & 752180.87 & 752837.21 & 0.087 & 5 & 1582228 \\
\hline 9 & & & & $0.10-3$ & 764681.13 & 766636.97 & 0.256 & 5 & 58313239 \\
\hline 10 & 4 & 1 & 0.6 & $0.10-5$ & 740258.05 & 753326.06 & 1.765 & 4 & 15631 \\
\hline 11 & & & & $0.10-7$ & 775601.31 & 793084.05 & 2.254 & 5 & 57303139 \\
\hline 12 & & & & $0.10-9$ & 722865.33 & 722865.33 & 0.000 & 5 & 58222839 \\
\hline 13 & & & & $0.05-0$ & 373154.06 & - & - & 3 & 5629 \\
\hline 14 & & & & $0.05-1$ & 373448.08 & 373981.80 & 0.143 & 3 & 52932 \\
\hline 15 & & & & $0.05-3$ & 369333.08 & 369333.22 & 0.000 & 3 & 5629 \\
\hline 16 & 5 & 1 & 0.2 & $0.05-5$ & 366591.17 & 368023.03 & 0.391 & 3 & 52931 \\
\hline 17 & & & & $0.05-7$ & 367453.19 & 367453.50 & 0.000 & 3 & 5629 \\
\hline 18 & & & & $0.05-9$ & 373457.30 & 375954.49 & 0.669 & 3 & 52932 \\
\hline $19^{\mathrm{a}}$ & & & & $0.01-5$ & 676227.20 & 676228.00 & 0.000 & 4 & 563139 \\
\hline $20^{\mathrm{a}}$ & & & & $0.02-5$ & 686512.72 & 688848.69 & 0.340 & 4 & 15631 \\
\hline $21^{\mathrm{a}}$ & & & & $0.05-5$ & 700426.72 & 700427.20 & 0.000 & 4 & 563139 \\
\hline $22^{\mathrm{b}}$ & 3 & 1 & 0.6 & $0.10-5$ & 747078.15 & 768003.33 & 2.801 & 4 & 15631 \\
\hline $23^{\mathrm{b}}$ & & & & $0.15-5$ & 810617.30 & 871868.94 & 7.556 & 5 & 45142829 \\
\hline $24^{\mathrm{b}}$ & & & & $0.20-5$ & 851678.43 & 950121.17 & 11.559 & 5 & 514223039 \\
\hline
\end{tabular}

${ }^{\mathrm{a}}$ For the corresponding identical- $q$ case, the number of DCs: 4, their locations: 563139 ;

${ }^{\mathrm{b}}$ For the corresponding identical- $q$ case, the number of DCs: 5, their locations: 58222839.

\subsection{Comparison to Other Models}

The LMRP generally results in optimal solutions that have fewer open facilities than those of the UFLP, due to the resulting economies of scale and risk-pooling effect that result from the inventory costs (Daskin et al. 2002, Shen et al. 2003). On the other hand, the RFLP tends to open more facilities than the UFLP, to encourage risk diversification (Snyder and Daskin 2005); a similar result holds for the HRFLP (RFLP with heterogeneous $q$ ). By incorporating both inventory and disruptions, our model can be viewed as finding a balance between these two competing tendencies. To demonstrate this, we solved each model for the 49-node instance with $\beta=1, \theta=2, L=1, R=4$. We assume that all DCs have the same failure probability (since our proposed LR approach is able to find near-optimal solutions for all instances in this test), which takes values ranging from 0 to 0.2 in increments of 0.01 


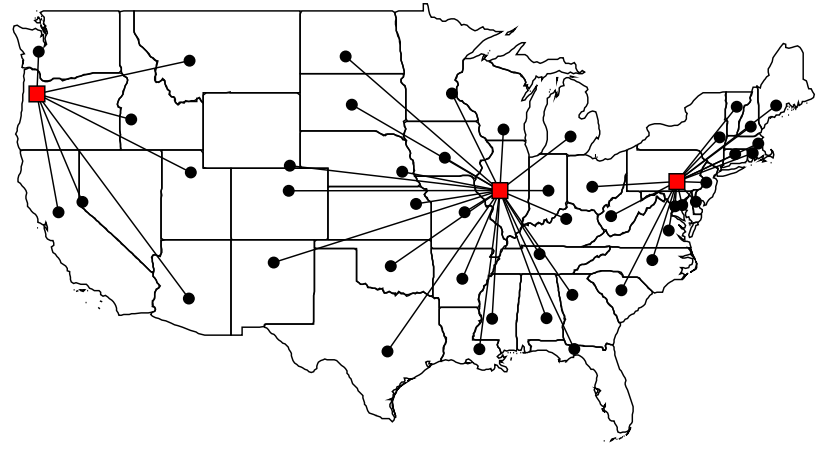

(a) $q=0.05-0,0.05-3,0.05-7$

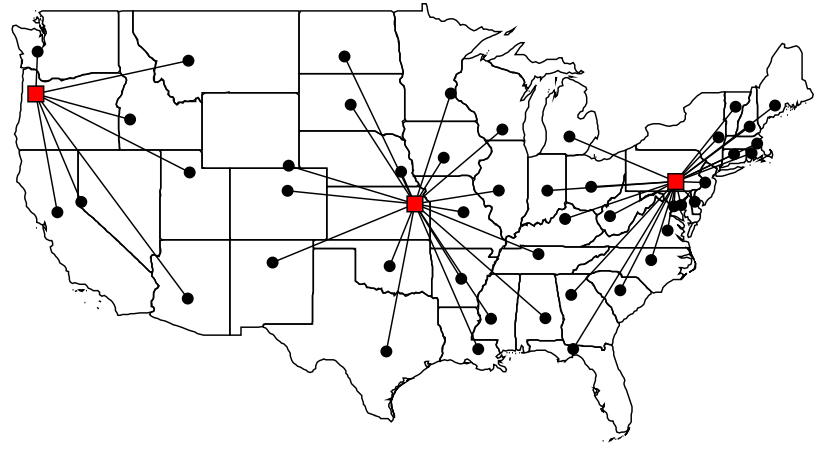

(b) $q=0.05-1,0.05-9$

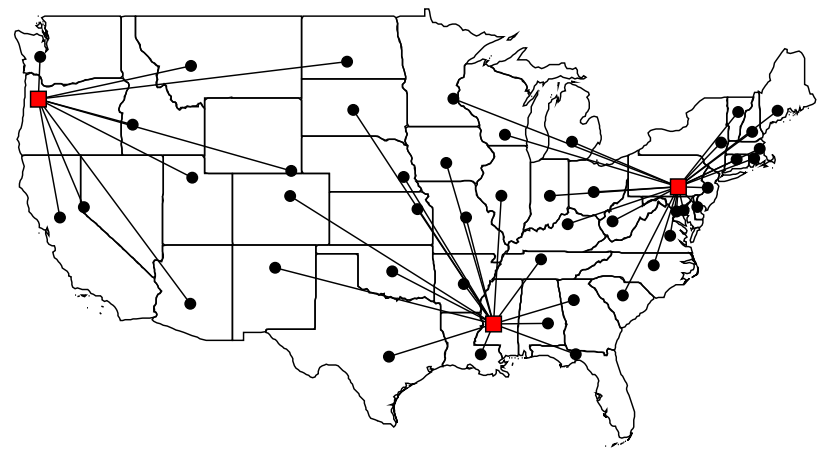

(c) $q=0.05-5$

Figure 2: 49 Nodes test $(R=5, L=1, \beta=0.2, E(q)=0.05)$

unit.

Figure 3 plots the number of open DCs as a function of the failure probability $q$. As expected, when $q=0$, the RLMRP is equivalent to the LMRP, while the RFLP is equivalent to the UFLP. As $q$ increases, the number of open DCs for the RFLP and the RLMRP increases, but the number for the RLMRP is never larger than that for the RFLP since the RLMRP also includes inventory costs. The fact that the RLMRP curve is closer to the RFLP curve than to the LMRP curve suggests that one makes a larger error by ignoring the disruption risk than by ignoring the inventory cost.

This claim is further supported by Figure 4, in which we plot the quality of the solutions to the UFLP, LMRP, and RFLP when evaluated under the RLMRP objective function, as $q$ changes. The solution quality is expressed as the percentage increase in cost vs. the optimal RLMRP cost. Clearly, ignoring disruptions (i.e., solving the LMRP) is much more costly than ignoring inventory (i.e., solving the RFLP). In fact, one would do better to ignore both disruptions and inventory (i.e., solving the UFLP) than to include inventory but not disruptions, since including inventory but not disruptions would bias the solution in the wrong direction. 


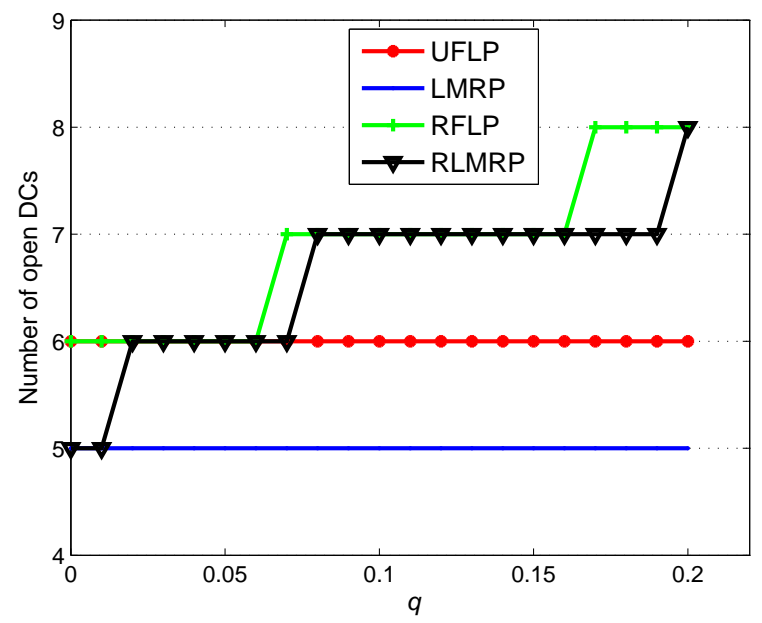

Figure 3: Number of open DCs for different $q$

The RFLP curve is quite close to the 0-line, suggesting that the RFLP can be a good heuristic, at least for these parameter settings. However, this may not be a safe approach to use, in general, since the RFLP model considers disruptions but not inventory. To show how the inventory cost term can influence the estimated accuracy, we change the weight of the inventory cost $\theta$ to take values in $\{2,4,6$, 8, 10\}; the results are illustrated in Figure 5. As we can see, the error increases as the inventory weight increases. For small failure probabilities (e.g., smaller than 0.1), the RFLP and RLMRP are both nearly equivalent to the UFLP since the disruption risk is low and the inventory costs are dominated by the transportation costs; therefore, there is 0 error from using the RFLP as a heuristic. For moderate $q$, the error from using the RFLP increases. Finally, if the failure probability is sufficiently large (e.g., larger than 0.2), then the impact of disruptions offsets the impact of inventory, and the RFLP is once again a good heuristic solution. This experiment suggests that, in general, it is important to consider the inventory cost, and the RFLP solution may not be a good heuristic solution. That is, except for some special cases, the UFLP, LMRP or RFLP solutions will not be accurate for the RLMRP, and instead one should use the proposed methods to solve the general problem.

\section{Conclusions and Future Research}

This paper proposes a reliable joint location-inventory model considering supply disruptions with heterogeneous disruption probabilities. This model determines the optimal number of facilities and their locations, the corresponding customer assignments, and inventory management policies that minimize 


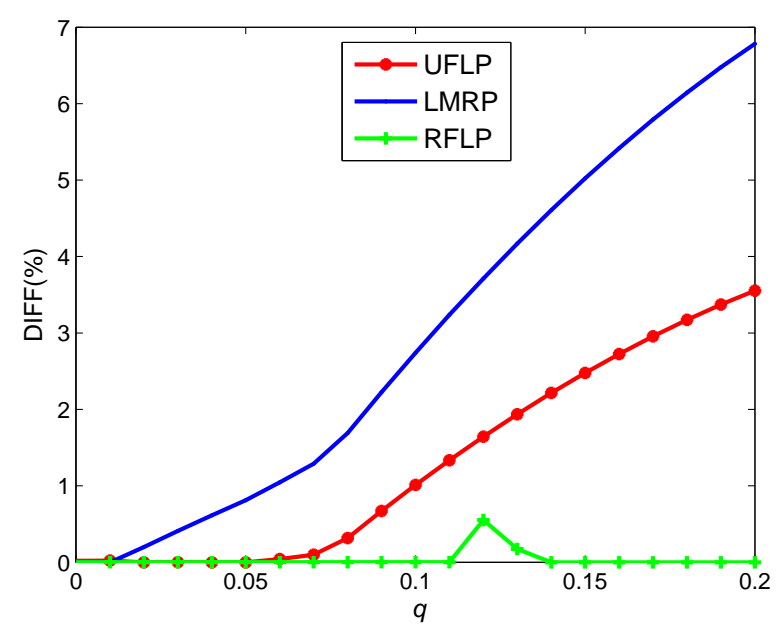

Figure 4: Objective values for different $q$

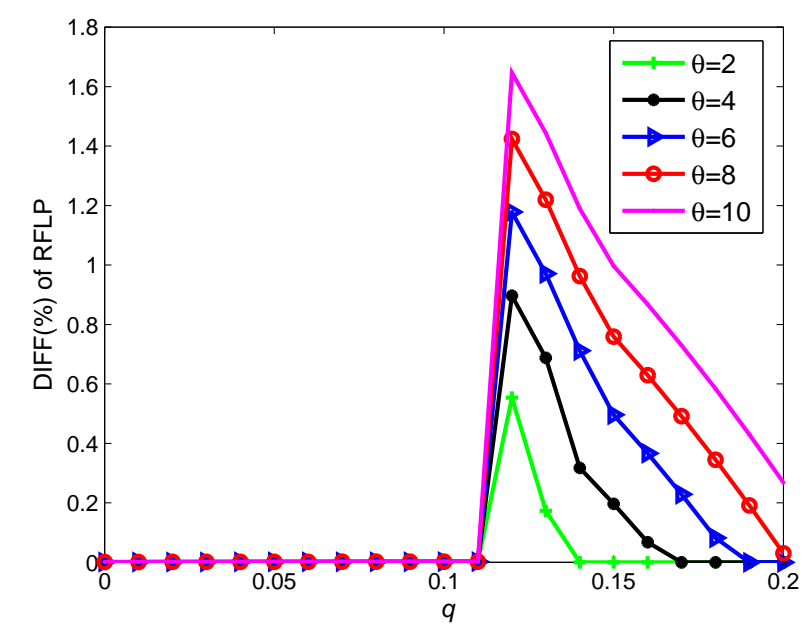

Figure 5: Objective values for different $q, \theta$ (RFLP)

facility setup costs, expected transportation costs and expected inventory holding costs across all possible facility disruption scenarios. Traditionally, because of the risk pooling effect and economies of scale, inventory costs tend to reduce the optimal number of open facilities; while because of the risk diversification effect, supply disruptions tend to result in more open facilities to reduce expected transportation costs. The model in this paper finds the optimal tradeoff between consolidation and diversification, by simultaneously considering the risk pooling effect and the possibility of facility failures.

We develop an exact as well as an approximate expression for the nonlinear inventory cost, under the assumption of heterogenous failure probabilities. Then we formulate a compact nonlinear mixed integer program, and design an exact approach using SOS2 and a heuristic using Lagrangian relaxation. Numerical results show that the proposed approach is able to obtain a good upper bound in a short time. Managerial insights about the problem are drawn from these results. For example, when the transportation cost dominates, customer demands will be spread to more facilities to reduce the shipment lengths; and when the inventory cost dominates, customer demands tend to be pooled together into fewer facilities. Moreover, savings in unmet-demand penalties can be reduced if customers can be assigned to multiple levels of backup facilities. In addition, replacing the heterogeneous disruption probabilities with their mean serves as a reasonable approximation for the heterogeneous model. On the other hand, ignoring either disruptions or inventory entirely (i.e., solving either the LMRP or the RFLP, respectively) can lead to large cost errors; if one must ignore one of these aspects, it is safer to ignore inventory and consider disruptions.

Our findings also bring up new questions for future research. First, we plan to extend the location- 
inventory model to allow spatially correlated disruptions, since it is generally observed that neighboring facilities are likely to fail simultaneously ( $\mathrm{Li}$ and Ouyang 2010). Correlations will bring new challenges, because the Lagrangian subproblem will no longer decompose by facility (Xie et al. 2015). Second, it would be useful to examine the optimal decisions in a dynamic environment, such as how the duration and the frequency of the facility disruptions would influence the assignment decisions. In this paper, we assume that if a facility fails, the customer must find an alternative available facility. However, in the real world, one can also simply wait until the disrupted facility becomes available again (Qi 2013$)$. As future research, we would like to find a suitable balance between these two strategies, waiting and dynamic routing. Finally, it is important to develop improvements to the standard branch-and-bound algorithm so that the gap can be closed without excessive branching, even under our substitution of constants in place of the probability variables.

\section{Acknowledgments}

This work is supported by the National Natural Science Foundation of China (71272030, 71502112), and partly sponsored by the State Scholarship Fund of China Scholarship Council. These supports are gratefully acknowledged. The authors thank Qi Chen and Yanfeng Ouyang for their help. We also wish to thank the anonymous referees for their helpful suggestions, which led to improved clarity of the paper.

\section{Appendix A Additional computational results}

Computational results for the HRLMRP on the 88-node dataset and the 150-node dataset, see Table A1 and Table A2.

\section{Appendix B Numerical Tests for Conjecture 1}

We conducted a numerical study to test Conjecture 1, as follows:

1. Generate 400 instances, consisting of each combination of $R \in\{2,3,4,5\}, E(q) \in\{0.01,0.05,0.10,0.15,0.20\}$, $n=5, \beta \in\{0.5,1.0,1.5,2.0,2.5\}$ and $\theta \in\{1,3,5,7\}$. For each instance, randomly choose a 20node subset of the 49-node dataset and set $I$ and $J$ both equal to this subset; randomly choose a subset of facilities $J^{\prime} \in J$, where $\left|J^{\prime}\right| \in\{2,3,4,5,6\}$. Each $\left|J^{\prime}\right|$ is used 10 times. This gives a total of 20,000 tests, which we call "partial solutions" since they choose facility locations but not 
Table A1: Numerical results for the 88-node dataset (HRLMRP, heterogenous $\boldsymbol{q}$ )

\begin{tabular}{|c|c|c|c|c|c|c|c|c|c|c|c|c|c|c|c|}
\hline \multirow[b]{2}{*}{ No. } & \multirow[b]{2}{*}{$R$} & \multirow[b]{2}{*}{$E(q)-n$} & \multirow[b]{2}{*}{$L$} & \multirow[b]{2}{*}{$\beta$} & \multirow{2}{*}{$\begin{array}{r}\text { Root } \\
\text { UB }\end{array}$} & \multirow{2}{*}{$\begin{array}{r}\text { Root } \\
\text { gap }\end{array}$} & \multirow[b]{2}{*}{ time } & \multirow[b]{2}{*}{$\mathrm{DC}$} & \multirow[b]{2}{*}{ In } & \multirow[b]{2}{*}{ Out } & \multirow{2}{*}{$\begin{array}{l}\text { Non- } \\
\text { closest }\end{array}$} & \multicolumn{4}{|c|}{ Cost component $(\%)$} \\
\hline & & & & & & & & & & & & Fixed & Inventory & Shipment & Penalty \\
\hline 1 & 1 & $0.01-5$ & 1 & 1.0 & 1705645.40 & 13.598 & 20.14 & 9 & 0 & 0 & 5 & 31.17 & 1.81 & 42.35 & 24.67 \\
\hline 2 & 1 & $0.05-5$ & 1 & 1.0 & 2733147.91 & 13.873 & 16.94 & 8 & 0 & 0 & 6 & 19.85 & 1.06 & 32.10 & 46.99 \\
\hline 3 & 1 & $0.10-5$ & 1 & 1.0 & 4188242.46 & 16.328 & 19.91 & 7 & 0 & 0 & 7 & 12.64 & 0.64 & 21.34 & 65.38 \\
\hline 4 & 1 & $0.15-5$ & 1 & 1.0 & 5631462.71 & 19.531 & 17.29 & 7 & 0 & 0 & 8 & 9.17 & 0.46 & 15.39 & 74.98 \\
\hline 5 & 1 & $0.20-5$ & 1 & 1.0 & 7042099.99 & 19.449 & 18.21 & 5 & 0 & 0 & 2 & 7.89 & 0.32 & 13.68 & 78.12 \\
\hline 6 & 5 & $0.01-5$ & 1 & 0.2 & 476014.14 & 0.254 & 22.31 & 4 & 0 & 83 & 0 & 45.21 & 4.53 & 50.27 & 0.00 \\
\hline 7 & 5 & $0.05-5$ & 1 & 0.2 & 495775.76 & 3.530 & 28.47 & 4 & 0 & 60 & 5 & 42.40 & 4.35 & 53.25 & 0.00 \\
\hline 8 & 5 & $0.10-5$ & 1 & 0.2 & 508399.82 & 2.705 & 37.99 & 4 & 0 & 70 & 5 & 41.35 & 4.24 & 54.37 & 0.04 \\
\hline 9 & 5 & $0.15-5$ & 1 & 0.2 & 561166.76 & 15.020 & 43.24 & 4 & 0 & 13 & 5 & 35.50 & 3.84 & 59.72 & 0.95 \\
\hline 10 & 5 & $0.20-5$ & 1 & 0.2 & 590195.16 & 18.083 & 44.32 & 4 & 0 & 7 & 6 & 39.67 & 3.65 & 55.67 & 1.02 \\
\hline 11 & 1 & $0.10-5$ & 1 & 0.6 & 2662083.74 & 15.186 & 22.54 & 5 & 0 & 0 & 3 & 14.93 & 0.87 & 23.82 & 60.39 \\
\hline 12 & 2 & $0.10-5$ & 1 & 0.6 & 1164604.27 & 11.860 & 25.24 & 5 & 0 & 0 & 16 & 23.76 & 2.05 & 63.47 & 10.72 \\
\hline 13 & 3 & $0.10-5$ & 1 & 0.6 & 1038865.77 & 6.823 & 34.16 & 7 & 0 & 15 & 29 & 37.66 & 2.70 & 57.86 & 1.79 \\
\hline 14 & 4 & $0.10-5$ & 1 & 0.6 & 1020616.62 & 5.263 & 44.23 & 6 & 0 & 0 & 0 & 30.22 & 2.54 & 67.09 & 0.15 \\
\hline 15 & 5 & $0.10-5$ & 1 & 0.6 & 1022363.20 & 5.446 & 41.65 & 7 & 0 & 19 & 31 & 38.16 & 2.74 & 59.08 & 0.02 \\
\hline 16 & 3 & $0.05-5$ & 1 & 0.2 & 496014.61 & 3.655 & 24.80 & 4 & 0 & 60 & 5 & 42.38 & 4.34 & 53.22 & 0.06 \\
\hline 17 & 3 & $0.05-5$ & 1 & 0.6 & 995659.94 & 6.227 & 30.17 & 6 & 0 & 17 & 0 & 31.74 & 2.61 & 65.23 & 0.43 \\
\hline 18 & 3 & $0.05-5$ & 1 & 1.0 & 1327575.79 & 4.310 & 30.17 & 9 & 0 & 1 & 4 & 37.95 & 2.35 & 59.31 & 0.39 \\
\hline 19 & 4 & $0.20-5$ & 1 & 0.2 & 590104.95 & 18.068 & 39.41 & 4 & 0 & 6 & 7 & 39.67 & 3.65 & 55.67 & 1.02 \\
\hline 20 & 4 & $0.20-5$ & 1 & 0.6 & 1148727.95 & 16.906 & 42.59 & 7 & 0 & 0 & 27 & 36.50 & 2.46 & 59.82 & 1.21 \\
\hline 21 & 4 & $0.20-5$ & 1 & 1.0 & 1544960.34 & 14.983 & 45.69 & 10 & 0 & 0 & 30 & 36.62 & 2.16 & 59.14 & 2.08 \\
\hline 22 & 3 & $0.20-5$ & 1 & 0.2 & 611825.02 & 19.843 & 30.70 & 4 & 0 & 6 & 7 & 38.26 & 3.51 & 52.78 & 5.44 \\
\hline 23 & 3 & $0.20-5$ & 3 & 0.2 & 613737.69 & 19.802 & 32.81 & 4 & 0 & 6 & 7 & 38.14 & 3.81 & 52.62 & 5.43 \\
\hline 24 & 3 & $0.20-5$ & 5 & 0.2 & 615054.56 & 19.774 & 32.85 & 4 & 0 & 6 & 7 & 38.06 & 4.02 & 52.51 & 5.41 \\
\hline 25 & 3 & $0.20-5$ & 7 & 0.2 & 616124.96 & 19.751 & 31.76 & 4 & 0 & 6 & 7 & 38.00 & 4.19 & 52.42 & 5.41 \\
\hline 26 & 3 & $0.20-5$ & 9 & 0.2 & 617050.52 & 19.731 & 34.68 & 4 & 0 & 6 & 7 & 37.94 & 4.33 & 52.34 & 5.40 \\
\hline Avg. & - & - & - & - & 1539135.39 & 12.692 & 31.24 & - & - & - & - & 32.49 & 2.82 & 49.40 & 15.29 \\
\hline
\end{tabular}

customer assignments. (We focus on 20-node instances since SOS2 can often solve instances of this size optimally in reasonable computational time.)

2. For each partial solution, fix facility locations to the set $J^{\prime}$, and solve the SOS2 formulation to optimize the assignments only, terminating either when the solver has found an optimal solution (to within a tolerance of $\varepsilon=0.1 \%$ ), or when a time limit of 600 seconds has been reached.

3. For each instance that was solved to optimality, identify all triplets $(i, j, k)$ for which customer $i$ is assigned to facility $k$ at level $r_{1}$ and to facility $j$ at level $r_{2}$ in the optimal solution, with $r_{1}<r_{2}$. For each such triplet, check inequality (34). If (34) holds, then we have found a counterexample to Conjecture 1, since (34) holds but it is still optimal to assign $i$ to $k$ at a lower level than to $j$.

In this experiment, 17,228 partial solutions were solved to optimality within the 600-second time limit. In total, the test evaluated $1,463,360$ triplets $(i, j, k)$ such that $i$ is assigned to facility $k$ at level $r_{1}$ and to facility $j$ at level $r_{2}$ in the optimal solutions, with $r_{1}<r_{2}$. (The number of such triplets is $20\left(\begin{array}{c}R \\ 2\end{array}\right)$ for an instance with 20 customers and maximum assignment level $R$.) For every one of these 
Table A2: Numerical results for the 150-node dataset (HRLMRP, heterogenous $\boldsymbol{q}$ )

\begin{tabular}{|c|c|c|c|c|c|c|c|c|c|c|c|c|c|c|c|}
\hline \multirow[b]{2}{*}{ No. } & \multirow[b]{2}{*}{$R$} & \multirow[b]{2}{*}{$E(q)-n$} & \multirow[b]{2}{*}{$L$} & \multirow[b]{2}{*}{$\beta$} & \multirow{2}{*}{$\begin{array}{r}\text { Root } \\
\mathrm{UB} \\
\end{array}$} & \multirow{2}{*}{$\begin{array}{r}\text { Root } \\
\text { gap } \\
\end{array}$} & \multirow[b]{2}{*}{ time } & \multirow[b]{2}{*}{$\mathrm{DC}$} & \multirow[b]{2}{*}{ In } & \multirow[b]{2}{*}{ Out } & \multirow{2}{*}{$\begin{array}{l}\text { Non- } \\
\text { closest }\end{array}$} & \multicolumn{4}{|c|}{ Cost component (\%) } \\
\hline & & & & & & & & & & & & Fixed & Inventory & Shipment & Penalty \\
\hline 1 & 1 & $0.01-5$ & 1 & 1.0 & 2126768.29 & 5.235 & 78.48 & 6 & 0 & 0 & 0 & 28.21 & 1.40 & 53.02 & 17.38 \\
\hline 2 & 1 & $0.05-5$ & 1 & 1.0 & 3427349.02 & 8.454 & 67.86 & 6 & 0 & 0 & 7 & 17.51 & 0.86 & 34.20 & 47.44 \\
\hline 3 & 1 & $0.10-5$ & 1 & 1.0 & 4954327.32 & 8.473 & 68.80 & 5 & 0 & 0 & 0 & 10.09 & 0.53 & 24.83 & 64.55 \\
\hline 4 & 1 & $0.15-5$ & 1 & 1.0 & 6900696.06 & 14.120 & 68.17 & 5 & 0 & 0 & 7 & 7.25 & 0.38 & 18.14 & 74.24 \\
\hline 5 & 1 & $0.20-5$ & 1 & 1.0 & 8582198.36 & 13.928 & 76.78 & 5 & 0 & 0 & 9 & 5.83 & 0.30 & 13.67 & 80.20 \\
\hline 6 & 5 & $0.01-5$ & 1 & 0.2 & 701684.23 & 0.560 & 73.82 & 3 & 0 & 123 & 0 & 42.75 & 3.03 & 54.22 & 0.00 \\
\hline 7 & 5 & $0.05-5$ & 1 & 0.2 & 729078.35 & 3.699 & 109.51 & 3 & 0 & 63 & 8 & 41.15 & 2.91 & 55.89 & 0.05 \\
\hline 8 & 5 & $0.10-5$ & 1 & 0.2 & 755594.24 & 5.793 & 150.09 & 4 & 0 & 15 & 1 & 52.94 & 3.25 & 43.80 & 0.01 \\
\hline 9 & 5 & $0.15-5$ & 1 & 0.2 & 795490.55 & 10.202 & 134.43 & 4 & 0 & 0 & 10 & 50.28 & 3.09 & 46.48 & 0.15 \\
\hline 10 & 5 & $0.20-5$ & 1 & 0.2 & 811555.15 & 11.174 & 130.32 & 4 & 0 & 0 & 17 & 49.29 & 3.03 & 47.41 & 0.28 \\
\hline 11 & 1 & $0.10-5$ & 1 & 0.6 & 3181496.56 & 7.573 & 67.63 & 4 & 0 & 0 & 4 & 12.57 & 0.75 & 26.23 & 60.45 \\
\hline 12 & 2 & $0.10-5$ & 1 & 0.6 & 1510115.25 & 7.563 & 79.11 & 5 & 0 & 0 & 9 & 33.11 & 1.79 & 57.68 & 7.42 \\
\hline 13 & 3 & $0.10-5$ & 1 & 0.6 & 1401125.12 & 5.773 & 103.65 & 6 & 0 & 0 & 22 & 42.82 & 2.13 & 54.34 & 0.72 \\
\hline 14 & 4 & $0.10-5$ & 1 & 0.6 & 1406195.85 & 6.375 & 156.06 & 6 & 0 & 0 & 43 & 42.67 & 2.10 & 55.18 & 0.04 \\
\hline 15 & 5 & $0.10-5$ & 1 & 0.6 & 1405730.43 & 6.358 & 162.55 & 6 & 0 & 0 & 43 & 42.68 & 2.10 & 55.21 & 0.00 \\
\hline 16 & 3 & $0.05-5$ & 1 & 0.2 & 728811.10 & 3.665 & 94.30 & 3 & 0 & 50 & 4 & 41.16 & 2.91 & 55.86 & 0.07 \\
\hline 17 & 3 & $0.05-5$ & 1 & 0.6 & 1349417.08 & 4.385 & 104.61 & 5 & 0 & 7 & 4 & 37.05 & 2.03 & 60.73 & 0.19 \\
\hline 18 & 3 & $0.05-5$ & 1 & 1.0 & 1837904.52 & 4.729 & 105.66 & 7 & 0 & 0 & 3 & 38.09 & 1.74 & 59.98 & 0.19 \\
\hline 19 & 4 & $0.20-5$ & 1 & 0.2 & 810803.99 & 11.091 & 114.57 & 4 & 0 & 0 & 15 & 49.33 & 3.03 & 47.36 & 0.28 \\
\hline 20 & 4 & $0.20-5$ & 1 & 0.6 & 1541081.40 & 15.133 & 110.28 & 5 & 0 & 0 & 26 & 32.45 & 1.76 & 65.40 & 0.39 \\
\hline 21 & 4 & $0.20-5$ & 1 & 1.0 & 2088027.46 & 15.582 & 106.66 & 9 & 0 & 1 & 65 & 43.10 & 1.76 & 54.24 & 0.90 \\
\hline 22 & 3 & $0.20-5$ & 1 & 0.2 & 831705.33 & 12.670 & 93.73 & 4 & 0 & 0 & 9 & 48.09 & 2.95 & 46.27 & 2.69 \\
\hline 23 & 3 & $0.20-5$ & 3 & 0.2 & 826549.00 & 11.917 & 96.13 & 4 & 0 & 0 & 17 & 48.39 & 3.24 & 46.14 & 2.23 \\
\hline 24 & 3 & $0.20-5$ & 5 & 0.2 & 836164.68 & 12.787 & 98.17 & 4 & 0 & 0 & 10 & 47.84 & 3.38 & 46.11 & 2.67 \\
\hline 25 & 3 & $0.20-5$ & 7 & 0.2 & 847358.99 & 13.825 & 108.97 & 3 & 0 & 0 & 8 & 35.40 & 3.00 & 59.32 & 2.27 \\
\hline 26 & 3 & $0.20-5$ & 9 & 0.2 & 833269.76 & 12.269 & 105.94 & 4 & 0 & 0 & 18 & 48.00 & 3.66 & 46.12 & 2.21 \\
\hline Avg. & - & - & - & - & 1970019.16 & 8.974 & 102.55 & - & - & - & - & 36.46 & 2.20 & 47.22 & 14.11 \\
\hline
\end{tabular}

triplets, inequality (34) is violated, i.e., there is no counterexample in the optimal solutions. In other words, this experiment supports our belief that Conjecture 1 is true.

We also solved each of the 20,000 partial solutions with a time limit of 10 seconds. The solver was able to solve 10,151 of them to optimality within 10 seconds, and could not find any feasible solution for another 352 partial solutions. An additional 1,775 partial solutions were not solved to provable optimality but still had no $(i, j, k)$ pairs that disprove the conjecture. For each of the remaining 7,722 partial solutions, we examined every triplet $(i, j, k)$ that satisfies (34) and for which $i$ is assigned to $k$ at a lower level than it is assigned to $j$. For each such triplet, we swapped the assignment levels for $k$ and $j$, and found that the resulting solution has a lower cost than the original solution. This lends further support to the truth of Conjecture 1.

Finally, we note that, among the 849,420 triplets $(i, j, k)$ in the 7,722 suboptimal solutions in the 10-second experiment, only seven triplets satisfied inequality (33) but not the stronger inequality (34). In other words, even if Conjecture 1 turns out to be false, the inequality in Proposition 2 is nearly as 
strong as that in Conjecture 1, and therefore is nearly as effective at identifying assignment triplets that cannot be optimal.

\section{Appendix C Proofs}

\section{C.1 Proof of Lemma 1}

Proof. Let $q_{[0]}^{\prime} \leq q_{[1]}^{\prime} \leq \cdots \leq q_{\left[\left|\mathcal{J}_{1}\right|-1\right]}^{\prime}$ and $q_{[0]}^{\prime \prime} \leq q_{[1]}^{\prime \prime} \leq \cdots \leq q_{\left[\left|\mathcal{J}_{2}\right|-1\right]}^{\prime \prime}$ be an ordering of the failure probabilities in $\mathcal{J}_{1}$ and $\mathcal{J}_{2}$, respectively. Then, by definition, we have $q_{[s]}^{\prime \prime} \leq q_{[s]}^{\prime}$ for $s=0,1, \cdots,\left|\mathcal{J}_{1}\right|-1$, which results in

$$
\prod_{s=0}^{r-1} q_{[s]}^{\prime \prime} \leq \prod_{s=0}^{r-1} q_{[s]}^{\prime}
$$

for $r=0,1, \cdots,\left|\mathcal{J}_{1}\right|$. Multiplying each side of this inequality by $\left(1-q_{j}\right)$, we obtain

$$
\left(1-q_{j}\right) \prod_{s=0}^{r-1} q_{[s]}^{\prime \prime} \leq\left(1-q_{j}\right) \prod_{s=0}^{r-1} q_{[s]}^{\prime},
$$

i.e., $Q_{j r}\left(\mathcal{J}_{1}\right) \geq Q_{j r}\left(\mathcal{J}_{2}\right)$. Similarly, $Q_{j r}\left(\mathcal{J}_{2}\right) \geq Q_{j r}\left(J_{e}\right)$.

Define $i[r] \in J_{e}$ to be the facility in $J_{e}$ that serves customer $i$ at level $r$, then

$$
p_{i j r}=\left(1-q_{j}\right) \prod_{s=0}^{r-1} q_{i[s]}
$$

From Aboolian et al. (2012), for a location set $\mathcal{J}_{1} \subseteq J_{e}$, we have $p_{i j r} \geq Q_{j r}\left(\mathcal{J}_{1}\right)$.

\section{C.2 Proof of Proposition 2}

Proof. Assume that $z_{i, k, r_{1}}=z_{i, j, r_{2}}=1$ for $r_{1}<r_{2}$. Without loss of generality, we can assume that $r_{2}=r_{1}+1$, since if $r_{2}>r_{1}+1$, then there must be some $\hat{r}_{1}$ and $\hat{r}_{2}, r_{1} \leq \hat{r}_{1}=\hat{r}_{2}-1<r_{2}$, such that the facility assigned to $i$ at level $\hat{r}_{1}$ is farther from $i$ than the facility assigned to $i$ at level $\hat{r}_{2}$ is.

Suppose we swap the assignments, so that $i$ is assigned to $j$ at level $r_{1}$ and to $k$ at level $r_{2}$. We will show this reduces the total cost.

Let $\boldsymbol{p}^{\prime}$ be the probability variables under the new solution (with $i$ 's assignments swapped). By (C1), since we do not change the assignments at levels $s=0, \cdots, r_{1}-1$ when we swap $i$ 's assignments, we have

$$
p_{i j r_{1}}^{\prime}=p_{i k r_{1}} \frac{1-q_{j}}{1-q_{k}}
$$


and

$$
p_{i k r_{2}}^{\prime}=p_{i j r_{2}} \frac{1-q_{k}}{1-q_{j}} \frac{q_{j}}{q_{k}}
$$

Moreover, since $r_{2}=r_{1}+1$,

$$
p_{i j r_{2}}=p_{i k r_{1}} q_{k} \frac{1-q_{j}}{1-q_{k}}
$$

Therefore,

$$
\begin{aligned}
p_{i j r_{1}}^{\prime}-p_{i j r_{2}} & =p_{i k r_{1}}\left(\frac{1-q_{j}}{1-q_{k}}-q_{k} \frac{1-q_{j}}{1-q_{k}}\right) \\
& =p_{i k r_{1}}\left(1-q_{j}\right) \\
& >0
\end{aligned}
$$

(The first equality follows from (C2) and (C4).) Similarly, we have

$$
\begin{aligned}
p_{i k r_{2}}^{\prime}-p_{i k r_{1}} & =p_{i k r_{1}}\left(\frac{1-q_{k}}{1-q_{j}} \frac{q_{j}}{q_{k}} \cdot q_{k} \frac{1-q_{j}}{1-q_{k}}-1\right) \\
& =p_{i k r_{1}}\left(q_{j}-1\right) \\
& <0
\end{aligned}
$$

Let $\Delta p=p_{i k r_{1}}\left(1-q_{j}\right)>0$. Note that $p_{i j r 1}^{\prime}-p_{i j r 2}=\Delta p$ and $p_{i k r 2}^{\prime}-p_{i k r 1}=-\Delta p$.

Below, we will use the following standard properties of the square-root function (or any concave function): If $0 \leq b \leq a$ and $c \geq 0$, then

$$
\begin{aligned}
& \frac{\sqrt{a}-\sqrt{a-b}}{b}>\frac{\sqrt{a+c}-\sqrt{a}}{c} \\
& \sqrt{a}-\sqrt{a-b}<\sqrt{b} \\
& \sqrt{b}-\sqrt{b+c}<\sqrt{a}-\sqrt{a+c} .
\end{aligned}
$$

The change in cost that results from swapping $i$ 's assignments is given by $\Delta_{j}+\Delta_{k}$, where $\Delta_{j}$ is the change in cost that results from assigning $i$ to $j$ at level $r_{1}$ instead of $r_{2}$, and $\Delta_{k}$ is the change in cost 
that results from assigning $i$ to $k$ at level $r_{2}$ instead of $r_{1}$. We have:

$$
\begin{aligned}
\Delta_{j}= & \hat{c}_{i j}\left(p_{i j r_{1}}^{\prime}-p_{i j r_{2}}\right) \\
& +\sqrt{\sum_{i^{\prime} \in I} \sum_{r=0}^{R-1} \hat{K}_{i^{\prime} j} p_{i^{\prime} j r} z_{i^{\prime} j r}-\hat{K}_{i j} p_{i j r_{2}}+\hat{K}_{i j} p_{i j r_{1}}^{\prime}}-\sqrt{\sum_{i^{\prime} \in I} \sum_{r=0}^{R-1} \hat{K}_{i^{\prime} j} p_{i^{\prime} j r} z_{i^{\prime} j r}} \\
= & \hat{c}_{i j}\left(p_{i j r_{1}}^{\prime}-p_{i j r_{2}}\right)+\sqrt{I C_{j}+\hat{K}_{i j}\left(p_{i j r_{1}}^{\prime}-p_{i j r_{2}}\right)}-\sqrt{I C_{j}} \\
= & \hat{c}_{i j} \Delta p+\sqrt{I C_{j}+\hat{K}_{i j} \Delta p}-\sqrt{I C_{j}} \\
< & \hat{c}_{i j} \Delta p+\frac{\hat{K}_{i j} \Delta p}{\hat{K}_{i j}}\left(\sqrt{I C_{j}}-\sqrt{I C_{j}-\hat{K}_{i j}}\right) \\
= & \hat{c}_{i j} \Delta p+\Delta p\left(\sqrt{I C_{j}}-\sqrt{I C_{j}-\hat{K}_{i j}}\right) \\
& <\Delta p\left(\hat{c}_{i j}+\sqrt{\hat{K}_{i j}}\right)
\end{aligned}
$$

where $I C_{j}=\sum_{i^{\prime} \in I} \sum_{r=0}^{R-1} \hat{K}_{i^{\prime} j} p_{i^{\prime} j r} z_{i^{\prime} j r}$. The first inequality follows from (C7) and the second from (C8), in each case setting $a=I C_{j}, b=\hat{K}_{i j}$, and $c=\hat{K}_{i j} \Delta p$.

Similarly,

$$
\begin{aligned}
\Delta_{k} & =\hat{c}_{i k}\left(p_{i k r_{2}}^{\prime}-p_{i k r_{1}}\right)+\sqrt{I C_{k}+\hat{K}_{i k}\left(p_{i k r_{2}}^{\prime}-p_{i k r_{1}}\right)}-\sqrt{I C_{k}} \\
& =-\hat{c}_{i k} \Delta p+\sqrt{I C_{k}-\hat{K}_{i k} \Delta p}-\sqrt{I C_{k}} \\
& <-\hat{c}_{i k} \Delta p+\frac{\hat{K}_{i k} \Delta p}{\hat{K}_{i k}}\left(\sqrt{I C_{k}}-\sqrt{I C_{k}+\hat{K}_{i k}}\right) \\
& =-\hat{c}_{i k} \Delta p+\Delta p\left(\sqrt{I C_{k}}-\sqrt{I C_{k}+\hat{K}_{i k}}\right) \\
& <\Delta p\left(-\hat{c}_{i k}+\sqrt{\sum_{i^{\prime} \in I} \hat{K}_{i^{\prime} k}}-\sqrt{\sum_{i^{\prime} \in I} \hat{K}_{i^{\prime} k}+\hat{K}_{i k}}\right)
\end{aligned}
$$

the first inequality results from (C7), setting $a=I C_{k}, b=\hat{K}_{i k} \Delta p$, and $c=\hat{K}_{i k}$. The second follows from (C9), setting $a=\sum_{i^{\prime} \in I} \hat{K}_{i^{\prime} k}, b=I C_{k}$, and $c=\hat{K}_{i k}$, noting that

$$
0 \leq b=I C_{k}=\sum_{i^{\prime} \in I} \sum_{r=0}^{R-1} \hat{K}_{i^{\prime} k} p_{i^{\prime} k r} z_{i^{\prime} k r} \leq a=\sum_{i^{\prime} \in I} \hat{K}_{i^{\prime} k}
$$

as required for (C9), since $\sum_{r=0}^{R-1} z_{i^{\prime} k r} \leq 1, \forall i^{\prime} \in I, k \in J$. Consequently, combining (C10) and (C11), we have that changing $i$ 's level- $r_{1}$ assignment from $k$ to $j$ and its level- $r_{2}$ assignment from $j$ to $k$ 
changes the objective function (6) by exactly

$$
\begin{aligned}
\Delta_{j}+\Delta_{k} & <\Delta p\left(\hat{c}_{i j}-\hat{c}_{i k}+\sqrt{\hat{K}_{i j}}+\sqrt{\sum_{i^{\prime} \in I} \hat{K}_{i^{\prime} k}}-\sqrt{\sum_{i^{\prime} \in I} \hat{K}_{i^{\prime} k}+\hat{K}_{i k}}\right) \\
& <0
\end{aligned}
$$

by assumption. Therefore, swapping the assignments for customer $i$ reduces the objective function value, and the original solution could not have been optimal.

\section{C.3 Proof of Lemma 2}

Proof. Define $\left(\boldsymbol{y}_{R P_{j}^{\prime}}^{*}, \boldsymbol{z}_{R P_{j}^{\prime}}^{*}\right)$ and $X_{R P_{j}^{\prime}}\left(\boldsymbol{y}_{R P_{j}^{\prime}}^{*}, z_{R P_{j}^{\prime}}^{*}\right)$ to be the optimal solution and optimal value of the objective function of $\left(\mathrm{RP}_{j}^{\prime}\right)$, respectively. Similarly, let $\left(\boldsymbol{y}_{R P_{j}}^{*}, z_{R P_{j}}^{*}\right)$ and $X_{R P_{j}}\left(\boldsymbol{y}_{R P_{j}}^{*}, \boldsymbol{z}_{R P_{j}}^{*}\right)$ be the optimal solution and optimal objective value of $\left(\mathrm{RP}_{j}\right)$, respectively.

Then by construction, $\left(\boldsymbol{y}_{R P_{j}}^{*}, \boldsymbol{z}_{R P_{j}}^{*}\right)$ is a feasible solution for $\left(\mathrm{RP}_{j}^{\prime}\right)$, and from Lemma 1 , we obtain

$$
X_{R P_{j}^{\prime}}\left(\boldsymbol{y}_{R P_{j}}^{*}, z_{R P_{j}}^{*}\right) \leq X_{R P_{j}}\left(\boldsymbol{y}_{R P_{j}}^{*}, z_{R P_{j}}^{*}\right)
$$

Also, because $\left(\boldsymbol{y}_{R P_{j}}^{*}, \boldsymbol{z}_{R P_{j}}^{*}\right)$ is the optimal solution of $\left(\mathrm{RP}_{j}\right)$, we have

$$
X_{R P_{j}^{\prime}}\left(\boldsymbol{y}_{R P_{j}^{\prime}}^{*}, z_{R P_{j}^{\prime}}^{*}\right) \leq X_{R P_{j}^{\prime}}\left(\boldsymbol{y}_{R P_{j}}^{*}, z_{R P_{j}}^{*}\right)
$$

Therefore, we conclude that

$$
X_{R P_{j}^{\prime}}\left(\boldsymbol{y}_{R P_{j}^{\prime}}^{*}, z_{R P_{j}^{\prime}}^{*}\right) \leq X_{R P_{j}}\left(\boldsymbol{y}_{R P_{j}}^{*}, z_{R P_{j}}^{*}\right)
$$

which implies that the optimal objective value of $\left(\mathrm{RP}_{j}^{\prime}\right)$ is a lower bound on that of the original $\left(\mathrm{RP}_{j}\right)$. Finally, from Aboolian et al. (2012), the optimal objective value of $\left(\mathrm{RP}_{j}\right)$ is a lower bound of (HRLMRP), which completes the proof.

\section{C.4 Other Proofs}

Lemma 3 Define two continuous functions $M_{i}(w)=\sqrt{w+\xi_{i}}-\sqrt{w}+\varphi_{i}, i=1,2$. If $\left(\varphi_{1}-\varphi_{2}\right)\left(M_{1}(0)-\right.$ $\left.M_{2}(0)\right)<0$, then the curves $M_{1}(w)$ and $M_{2}(w)$ intersect at

$$
w=\frac{1}{4}\left(\varphi_{2}-\varphi_{1}+\frac{\xi_{1}-\xi_{2}}{\varphi_{2}-\varphi_{1}}\right)^{2}-\xi_{1}=\frac{1}{4}\left(\varphi_{2}-\varphi_{1}-\frac{\xi_{1}-\xi_{2}}{\varphi_{2}-\varphi_{1}}\right)^{2}-\xi_{2}
$$


Proof. Let $M_{1}(w)=M_{2}(w)$. Then

$$
\begin{aligned}
M_{1}(w)=M_{2}(w) & \Longrightarrow \sqrt{w+\xi_{1}}-\sqrt{w}+\varphi_{1}=\sqrt{w+\xi_{2}}-\sqrt{w}+\varphi_{2} \\
& \Longrightarrow \sqrt{w+\xi_{1}}+\varphi_{1}=\sqrt{w+\xi_{2}}+\varphi_{2} \\
& \Longrightarrow \sqrt{w+\xi_{1}}-\sqrt{w+\xi_{2}}=\varphi_{2}-\varphi_{1} \\
& \Longrightarrow \xi_{1}-\xi_{2}=\left(\varphi_{2}-\varphi_{1}\right)\left(\sqrt{w+\xi_{1}}+\sqrt{w+\xi_{2}}\right) \\
& \Longrightarrow \sqrt{w+\xi_{1}}+\sqrt{w+\xi_{2}}=\frac{\xi_{1}-\xi_{2}}{\varphi_{2}-\varphi_{1}}
\end{aligned}
$$

Summing (C12) and (C13), we get

$$
w=\frac{1}{4}\left(\varphi_{2}-\varphi_{1}+\frac{\xi_{1}-\xi_{2}}{\varphi_{2}-\varphi_{1}}\right)^{2}-\xi_{1}
$$

Subtracting (C12) from (C13), we get

$$
w=\frac{1}{4}\left(\frac{\xi_{1}-\xi_{2}}{\varphi_{2}-\varphi_{1}}+\varphi_{1}-\varphi_{2}\right)^{2}-\xi_{2}=\frac{1}{4}\left(\varphi_{2}-\varphi_{1}-\frac{\xi_{1}-\xi_{2}}{\varphi_{2}-\varphi_{1}}\right)^{2}-\xi_{2}
$$

Lemma 4 Define three continuous functions $M_{i}(w)=\sqrt{w+\xi_{i}}-\sqrt{w}+\varphi_{i}, i=1,2,3$, with domain $[0, \infty)$. Denote the intersection of curves $M_{i}(w)$ and $M_{j}(w)$ as $\bar{w}_{i j}, i \neq j \in\{1,2,3\}$. Let $\mathcal{F}_{i j}=$ $\varphi_{i}+\frac{\xi_{i}-\xi_{j}}{\varphi_{j}-\varphi_{i}}$. Suppose $\varphi_{1}>\varphi_{2}>\varphi_{3}$ and $M_{1}(0)<M_{2}(0)<M_{3}(0)$.

(1) $\mathcal{F}_{12} \geq \mathcal{F}_{32}$ if and only if $\bar{w}_{12} \geq \bar{w}_{23}$.

(2) If $\mathcal{F}_{12}<\mathcal{F}_{32}$, then $\bar{w}_{12}<\bar{w}_{13}<\bar{w}_{23}$.

Proof. Note that $M_{1}(0)=\sqrt{\xi_{1}}+\varphi_{1}<M_{2}(0)=\sqrt{\xi_{2}}+\varphi_{2}<M_{3}(0)=\sqrt{\xi_{3}}+\varphi_{3}$; then since $\varphi_{1}>\varphi_{2}>\varphi_{3}$, we must have $\xi_{1}<\xi_{2}<\xi_{3}$. Furthermore,

$$
\begin{aligned}
M_{2}(0)<M_{3}(0) & \Longrightarrow \sqrt{\xi_{2}}+\varphi_{2}<\sqrt{\xi_{3}}+\varphi_{3} \\
& \Longrightarrow \sqrt{\xi_{2}}-\sqrt{\xi_{3}}<\varphi_{3}-\varphi_{2} \\
& \Longrightarrow \xi_{2}-\xi_{3}<\left(\varphi_{3}-\varphi_{2}\right)\left(\sqrt{\xi_{2}}+\sqrt{\xi_{3}}\right) \\
& \Longrightarrow \frac{\xi_{2}-\xi_{3}}{\varphi_{3}-\varphi_{2}}>\sqrt{\xi_{2}}+\sqrt{\xi_{3}} \\
& \Longrightarrow \varphi_{3}-\varphi_{2}+\frac{\xi_{2}-\xi_{3}}{\varphi_{3}-\varphi_{2}}>\varphi_{3}-\varphi_{2}+\sqrt{\xi_{2}}+\sqrt{\xi_{3}}>\sqrt{\xi_{2}}+\varphi_{2}+\sqrt{\xi_{2}}-\varphi_{2}=2 \sqrt{\xi_{2}}>0
\end{aligned}
$$

(The last inequality follows from the fact that $\sqrt{\xi_{3}}+\varphi_{3}>\sqrt{\xi_{2}}+\varphi_{2}$.) Therefore, $\varphi_{3}-\varphi_{2}+\frac{\xi_{2}-\xi_{3}}{\varphi_{3}-\varphi_{2}}>0$.

Furthermore, since $\xi_{1}<\xi_{2}$ and $\varphi_{1}>\varphi_{2}$, clearly $\varphi_{1}-\varphi_{2}+\frac{\xi_{1}-\xi_{2}}{\varphi_{2}-\varphi_{1}}>0$. 
(1) By Lemma 3, $\bar{w}_{12}=\frac{1}{4}\left(\varphi_{1}-\varphi_{2}+\frac{\xi_{1}-\xi_{2}}{\varphi_{2}-\varphi_{1}}\right)^{2}-\xi_{2}$ and $\bar{w}_{23}=\frac{1}{4}\left(\varphi_{3}-\varphi_{2}+\frac{\xi_{2}-\xi_{3}}{\varphi_{3}-\varphi_{2}}\right)^{2}-\xi_{2}$. By assumption,

$$
\begin{aligned}
\mathcal{F}_{12} \geq \mathcal{F}_{32} & \Longrightarrow \varphi_{1}-\varphi_{2}+\frac{\xi_{1}-\xi_{2}}{\varphi_{2}-\varphi_{1}} \geq \varphi_{3}-\varphi_{2}+\frac{\xi_{2}-\xi_{3}}{\varphi_{3}-\varphi_{2}} \\
& \Longrightarrow\left(\varphi_{1}-\varphi_{2}+\frac{\xi_{1}-\xi_{2}}{\varphi_{2}-\varphi_{1}}\right)^{2} \geq\left(\varphi_{3}-\varphi_{2}+\frac{\xi_{2}-\xi_{3}}{\varphi_{3}-\varphi_{2}}\right)^{2} \\
& \Longrightarrow \bar{w}_{12} \geq \bar{w}_{23} .
\end{aligned}
$$

Conversely,

$$
\begin{aligned}
\bar{w}_{12} \geq \bar{w}_{23} & \Longrightarrow \varphi_{1}-\varphi_{2}+\frac{\xi_{1}-\xi_{2}}{\varphi_{2}-\varphi_{1}} \geq \varphi_{3}-\varphi_{2}+\frac{\xi_{2}-\xi_{3}}{\varphi_{3}-\varphi_{2}} \\
& \Longrightarrow \varphi_{1}+\frac{\xi_{1}-\xi_{2}}{\varphi_{2}-\varphi_{1}} \geq \varphi_{3}+\frac{\xi_{2}-\xi_{3}}{\varphi_{3}-\varphi_{2}} \\
& \Longrightarrow \mathcal{F}_{12} \geq \mathcal{F}_{32}
\end{aligned}
$$

Therefore, $\mathcal{F}_{12} \geq \mathcal{F}_{32} \Longleftrightarrow \bar{w}_{12} \geq \bar{w}_{23}$.

(2) From (1), we know that $\bar{w}_{12}<\bar{w}_{23}$. Figure C1 shows the three possible cases for the intersections of these three curves: (a) $\bar{w}_{23}<\bar{w}_{13}<\bar{w}_{12}$; (b) $\bar{w}_{12}=\bar{w}_{13}=\bar{w}_{23}$; (c) $\bar{w}_{12}<\bar{w}_{13}<\bar{w}_{23}$. (a) corresponds the case that $\mathcal{F}_{12}>\mathcal{F}_{32}$ and $\bar{w}_{12}>\bar{w}_{23}$. (b) corresponds the case that these three curves have the same intersection. (c) corresponds to the case that $\mathcal{F}_{12}<\mathcal{F}_{32}$ and $\bar{w}_{12}<\bar{w}_{23}$. Since only these three cases exist, under the given condition, we must have $\bar{w}_{12}<\bar{w}_{13}<\bar{w}_{23}$.

\section{Appendix D A counterexample to the partition in Chen et al. (2011)}

The definitions of intervals $\left[w_{k}^{i-}, w_{k}^{i+}\right], k=0, \cdots,\left|N_{i}\right|-1$ does not guarantee a non-overlapping partition of $[0, \infty)$ in some situations. Table D1 provides parameter values for a simple example for a given $i$ for $r=0,1,2$. By definition, $R_{i}=N_{i}=\{0,1,2\}$, and the elements of $N_{i}$ are sorted into $r(i, 0)=$ $2, r(i, 1)=1, r(i, 2)=0$. The three continuous functions $M_{i r}(w)=\sqrt{w+\xi_{i r}}-\sqrt{w}+\varphi_{i r}, r=0,1,2$, have three intersections: $\bar{w}_{12}^{i}=\bar{w}_{21}^{i}=11.25, \bar{w}_{02}^{i}=\bar{w}_{20}^{i}=8$, and $\bar{w}_{10}^{i}=\bar{w}_{01}^{i}=3.25$. Therefore,

$$
\begin{aligned}
& w_{0}^{i-}=0 \\
& w_{0}^{i+}=\min \left\{\bar{w}_{21}^{i}, \bar{w}_{20}^{i}\right\}=8 \\
& w_{1}^{i-}=\max \left\{\bar{w}_{12}^{i}\right\}=11.25 \\
& w_{1}^{i+}=\min \left\{\bar{w}_{10}^{i}\right\}=3.25 \\
& w_{2}^{i-}=\max \left\{\bar{w}_{01}^{i}, \bar{w}_{02}^{i}\right\}=8 \\
& w_{2}^{i+}=\infty .
\end{aligned}
$$

Obviously, interval $\left[w_{1}^{-}, w_{1}^{+}\right]$is empty since $w_{1}^{-}>w_{1}^{+}$. 


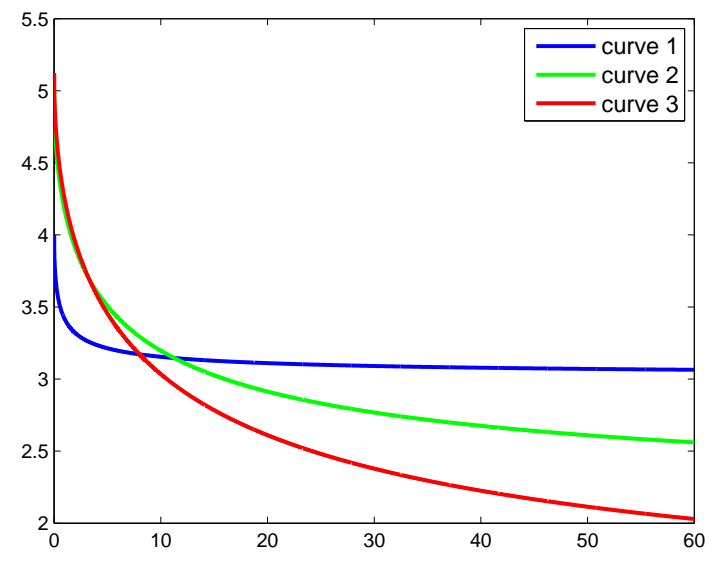

(a)

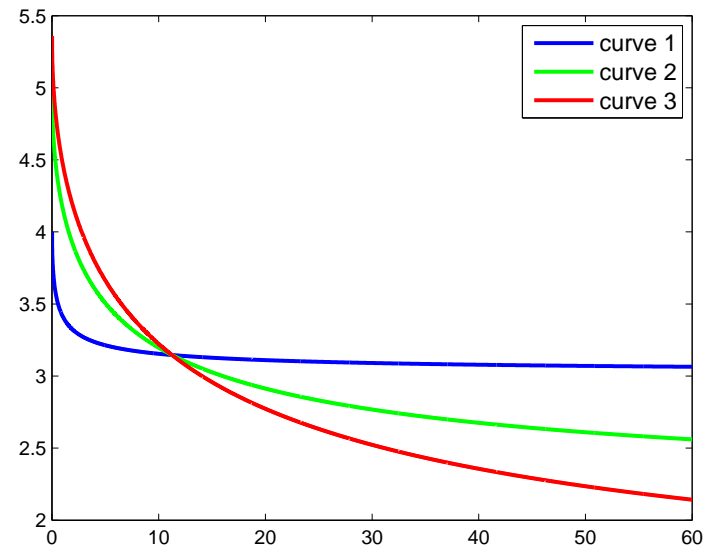

(b)

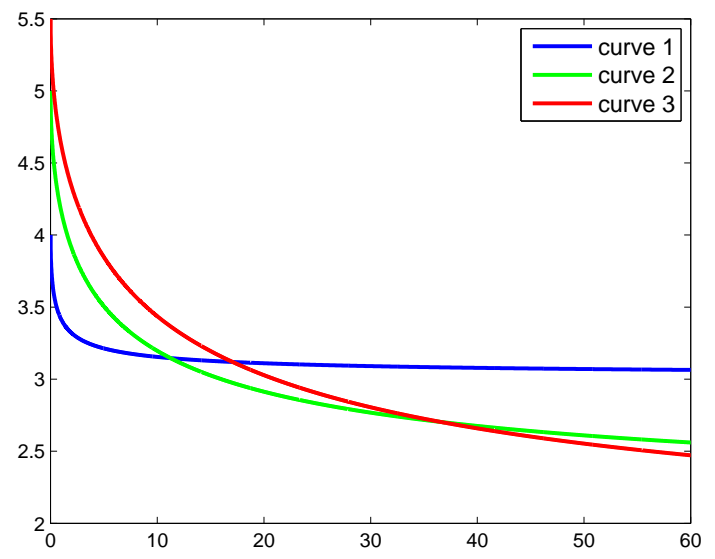

(c)

Figure C1: Insersections of the three curves

Table D1: Counterexample to partitioning-intervals claim

\begin{tabular}{cccc}
\hline & $r=0$ & $r=1$ & $r=2$ \\
\hline$\xi_{i r}$ & 17 & 9 & 1 \\
$\varphi_{i r}$ & 1 & 2 & 3 \\
$M_{i r}(0)=\sqrt{\xi_{i r}}+\varphi_{i r}$ & 5.1231 & 5 & 4 \\
\hline
\end{tabular}

\section{References}

Aboolian, Robert, Tingting Cui, Zuo-Jun Max Shen. 2012. An efficient approach for solving reliable facility location models. INFORMS Journal on Computing 25(4) 720-729.

Aryanezhad, Mir-Bahador, Seyed Gholamreza Jalali, Armin Jabbarzadeh. 2010. An integrated supply chain 
design model with random disruptions consideration. African Journal of Business Management 4(12) 2393-2401.

Berman, Oded, Dmitry Krass, Mozart BC Menezes. 2007. Facility reliability issues in network p-median problems: strategic centralization and co-location effects. Operations Research 55(2) 332-350.

Chen, Qi, Xiaopeng Li, Yanfeng Ouyang. 2011. Joint inventory-location problem under the risk of probabilistic facility disruptions. Transportation Research Part B: Methodological 45(7) 991-1003.

Cui, Tingting, Yanfeng Ouyang, Zuo-Jun Max Shen. 2010. Reliable facility location design under the risk of disruptions. Operations Research 58(4-part-1) 998-1011.

Daskin, Mark S. 2013. Network and Discrete Location: Models, Algorithms, and Applications. 2nd ed. Wiley, New York.

Daskin, Mark S, Collette R Coullard, Zuo-Jun Max Shen. 2002. An inventory-location model: Formulation, solution algorithm and computational results. Annals of Operations Research 110(1-4) 83-106.

Eppen, Gary D. 1979. Note-effects of centralization on expected costs in a multi-location newsboy problem. Management Science 25(5) 498-501.

Jeon, Hyong-Mo. 2008. Location-inventory models with supply disruptions. Ph.D. thesis, Lehigh University.

Li, Xiaopeng, Yanfeng Ouyang. 2010. A continuum approximation approach to reliable facility location design under correlated probabilistic disruptions. Transportation Research Part B: Methodological 44(4) 535-548.

Luedtke, James, Mahdi Namazifar, Jeff Linderoth. 2012. Some results on the strength of relaxations of multilinear functions. Mathematical programming 136(2) 325-351.

Maass, Kayse Lee, Mark S Daskin, Siqian Shen. 2015. Mitigating hard capacity constraints with inventory in facility location modeling. IIE Transactions, forthcoming .

McCormick, Garth P. 1976. Computability of global solutions to factorable nonconvex programs: Part I—convex underestimating problems. Mathematical programming 10(1) 147-175.

Ozsen, Leyla, Collette R Coullard, Mark S Daskin. 2008. Capacitated warehouse location model with risk pooling. Naval Research Logistics (NRL) 55(4) 295-312.

Ozsen, Leyla, Mark S Daskin, Collette R Coullard. 2009. Facility location modeling and inventory management with multisourcing. Transportation Science 43(4) 455-472.

Qi, Lian. 2013. A continuous-review inventory model with random disruptions at the primary supplier. European Journal of Operational Research 225(1) 59-74.

Qi, Lian, Zuo-Jun Max Shen. 2007. A supply chain design model with unreliable supply. Naval Research Logistics (NRL) 54(8) 829-844.

Qi, Lian, Zuo-Jun Max Shen, Lawrence V Snyder. 2010. The effect of supply disruptions on supply chain design decisions. Transportation Science 44(2) 274-289. 
Shen, Zuo-Jun Max, Collette Coullard, Mark S Daskin. 2003. A joint location-inventory model. Transportation Science 37(1) 40-55.

Shen, Zuo-Jun Max, Lian Qi. 2007. Incorporating inventory and routing costs in strategic location models. European Journal of Operational Research 179(2) 372-389.

Shen, Zuo-Jun Max, Roger Lezhou Zhan, Jiawei Zhang. 2011. The reliable facility location problem: Formulations, heuristics, and approximation algorithms. INFORMS Journal on Computing 23(3) 470-482.

Sherali, Hanif D, Amine Alameddine. 1992. A new reformulation-linearization technique for bilinear programming problems. Journal of Global Optimization 2(4) 379-410.

Shu, Jia, Chung-Piaw Teo, Zuo-Jun Max Shen. 2005. Stochastic transportation-inventory network design problem. Operations Research 53(1) 48-60.

Snyder, Lawrence V., Zümbül Atan, Peng Peng, Ying Rong, Amanda Schmitt, Burcu Sinsoysal. 2015. OR/MS models for supply chain disruptions: A review. IIE Transactions, forthcoming .

Snyder, Lawrence V, Mark S Daskin. 2005. Reliability models for facility location: the expected failure cost case. Transportation Science 39(3) 400-416.

Snyder, Lawrence V., Zuo-Jun Max Shen. 2011. Fundamentals of Supply Chain Theory. Wiley.

Xie, Siyang, Xiaopeng Li, Yanfeng Ouyang. 2015. Decomposition of general facility disruption correlations via augmentation of virtual supporting stations. Transportation Research Part B: Methodological 80 64-81. 